\title{
1 BubR1 recruitment to the kinetochore via Bub1 enhances Spindle Assembly Checkpoint \\ 2 signaling
}

3 Anand Banerjee ${ }^{1, *}, \#$, Chu Chen ${ }^{2,}$, , Lauren Humphrey ${ }^{2}$, John J. Tyson $^{1}$, Ajit P. Joglekar ${ }^{2, \#}$

41 - Academy of Integrated Science, Virginia Polytechnic Institute \& State University, Blacksburg,

5 VA 24601, USA

62 - Cell \& Developmental Biology, University of Michigan Medical School, Ann Arbor, MI 48109,

7 USA

$8 \quad$ * - These authors contributed equally.

$9 \quad \#$ - corresponding authors

\section{Abstract}

11 During mitosis, unattached kinetochores in a dividing cell generate the anaphase-inhibitory Mitotic

12 Checkpoint Complex (MCC) to activate the Spindle Assembly Checkpoint (SAC) and delay

13 anaphase onset. To generate MCC, these kinetochores recruit MCC constituent proteins

14 including the protein BubR1. The increased local concentration of BubR1 resulting from this

15 recruitment should enhance MCC generation, but prior studies found this not to be the case. We

16 analyzed the contribution of two BubR1 recruitment pathways to MCC generation in human

17 kinetochores. For these analyses, we isolated a subset of the MCC generation reactions to the

18 cytosol using ectopic SAC activation systems. These analyses and mathematical modeling show

19 that BubR1 binding to the SAC protein Bub1, but not to the 'KI' motifs in the kinetochore protein

$20 \mathrm{Knl}$, significantly enhances the rate of Bub1-mediated MCC generation in the kinetochore. Our

21 work also suggests that Bub1-BubR1 stoichiometry will strongly influence the dose-response

22 characteristics of SAC signaling.

23

24 Keywords: mitosis, Spindle Assembly Checkpoint, eSAC, kinetochores, mathematical modeling 


\section{Introduction}

26 The "Spindle Assembly Checkpoint" (SAC) is a cell cycle control that minimizes chromosome

27 missegregation during cell division (Musacchio, 2015). It is activated by kinetochores that are not

28 stably attached to the plus-ends of spindle microtubules. These kinetochores generate a diffusible

29 anaphase-inhibitory signal in the form of the Mitotic Checkpoint Complex (MCC), which in turn

30 delays anaphase onset to avert cell division. The amount of MCC in the dividing cell controls the

31 delay in anaphase onset. This amount depends on the number of unattached kinetochores and

32 the rate at which each kinetochore generates MCC (Collin et al., 2013; Dick and Gerlich, 2013).

33 Therefore, the latter parameter is a key determinant of the delay in anaphase onset and

34 chromosome segregation accuracy (Roy et al., 2020).

35 The rate at which an unattached kinetochore generates MCC depends on its ability to recruit MCC 36 components Mad2, and Cdc20 (Vleugel et al., 2015; Vleugel et al., 2013) (Figure 1A, dashed

37 square). Upon their recruitment to unattached kinetochores, these proteins interact to generate 38 either the MCC itself (or the C-Mad2-Cdc20 subcomplex). Recent discoveries show that Bub1 39 plays a major role in coordinating the formation of the C-Mad2-Cdc20 subcomplex, which is a 40 crucial, but rate-limiting step in MCC formation (Faesen et al., 2017; Lara-Gonzalez et al., 2021;

41 Piano et al., 2021). Bub1 also heterodimerizes with BubR1 (Overlack et al., 2015). Given this

42 knowledge, it is reasonable to expect that BubR1 recruitment to the kinetochore will increase the 43 rate of MCC generation. Interestingly, however, this expectation does not appear to hold.

44 Disruption of BubR1 recruitment to unattached kinetochores does not reduce the duration of SAC45 induced mitotic arrest in nocodazole-treated cells (Overlack et al., 2015; Zhang et al., 2016). This 46 finding is counter-intuitive, because decreased local BubR1 concentration in the kinetochore 47 should reduce the rate of MCC formation by the kinetochore, and therefore lessen the delay in 48 anaphase onset. A possible explanation for this finding is that BubR1 recruits Protein 49 Phosphatase 2A (PP2A) to the kinetochore, which indirectly promotes SAC silencing (Foley et 50 al., 2011; Nijenhuis et al., 2014).

51 In this study, we re-examine the contribution of kinetochore recruitment of BubR1 to SAC 52 signaling in HeLa cells. To overcome the challenges associated with studying SAC signaling 53 within kinetochores, we use the ectopic SAC activation system or the 'eSAC' to isolate specific 54 signaling interactions in the cytosol (Chen et al., 2019). We find that Bub1-BubR1 55 heterodimerization elevates Bub1-mediated MCC generation. On the other hand, the recruitment 56 of Bub1 and BubR1 via the 'Kl' motifs in the kinetochore protein Knl1 does not contribute to MCC 57 generation mediated by the MELT motifs within the Knl1 phosphodomain (Bolanos-Garcia et al., 
2011a; Bolanos-Garcia et al., 2011b; Kiyomitsu et al., 2011; Krenn et al., 2012; Primorac et al., 2013; Vleugel et al., 2013; Zhang et al., 2014). We also establish a mathematical model to elucidate the mechanistic details of the SAC signaling cascade that generates the MCC. We confirm these findings in the context of kinetochore-based SAC signaling.

\section{Results}

\section{The BubR1 binding domain of Bub1 promotes Bub1-mediated MCC assembly}

BubR1 is recruited to the human kinetochore by: (1) its heterodimerization with Bub1, which itself binds to the kinetochore protein $\mathrm{Knl} 1$ as part of the Bub1-Bub3 complex, and (2) its binding to the 'KI motif' located within the N-terminal domain of Knl1 (Figure 1A) (Bolanos-Garcia et al., 2011b; Kiyomitsu et al., 2011; Krenn et al., 2014; Leontiou et al., 2019; Nijenhuis et al., 2013). We first examined whether Bub1-BubR1 heterodimerization contributes to Bub1-mediated MCC assembly. Conventional assays are ill-suited to directly address this question, because both Bub1 and BubR1 recruit regulatory enzymes to the kinetochore. Consequently, any disruption of the recruitment of the SAC proteins to the kinetochore is bound to perturb the intricate regulation of signaling activities within the kinetochore. Therefore, we first decided to employ the ectopic SAC activation system (the eSAC) to isolate Bub1-mediated MCC generation in the cytoplasm and then quantitatively dissect the role of Bub1-BubR1 heterodimerization in MCC assembly.

We and others have previously found that the induced dimerization of a fragment of the central domain of Bub1 with Mps1 delays anaphase onset in HeLa cells, budding yeast, and fission yeast (Chen et al., 2019; Leontiou et al., 2019; Yuan et al., 2017). In HeLa cells, induced dimerization of the central domain of Bub1 (Bub1 ${ }^{231-620}-\mathrm{mNG}-2 x F k b p 12$, diagram in Figure 1B) with the Mps1 kinase domain (Frb-mCherry-Mps $1^{500-817}$ ) produced a dose-dependent delay in anaphase onset with a maximal duration of $\sim 145$ minutes (Figure 1B replotted for comparison from (Chen et al., 2019)). To test whether Bub1-BubR1 heterodimerization contributes to Bub1-mediated MCC assembly, we performed dose-response analysis on a truncated Bub1 phosphodomain that lacks the BubR1 heterodimerization domain (Bub1 ${ }^{444-620}$-mNG-2xFkbp12) (Overlack et al., 2015). Ectopic SAC activation using this truncated Bub1 phosphodomain elicited a significantly weaker response, with a maximal mitotic duration of $\sim 105$ minutes (Figure $1 \mathrm{C}$, plateau of the 4-parameter sigmoid predicted as $105 \pm 6$ minutes, the range indicates $95 \%$ confidence intervals, see Materials and Methods for details). It should be noted that the removal of the Bub3-binding GLEBS domain 
mitotic delay induced by the eSAC activity (Roy et al., 2021). Thus, Bub1-BubR1

91 heterodimerization contributes to the ability of the Bub1 phosphodomain to generate MCC. A

92 similar observation was reported by a recent in vitro study (Piano et al., 2021).

93 To understand whether a mechanism based on local BubR1 enrichment can explain the dose-

94 response data, we constructed a numerical model that simulates key steps in MCC generation by

95 the eSAC system and the effect of this MCC on the metaphase-to-anaphase transition (see

96 Materials and Methods). This model consists of two stages. In the first stage, we calculate the

97 equilibrium concentrations of two types of signaling complexes that the Bub1 phosphodomain

98 (Bub1 $\left.{ }^{231-620}\right)$ can form: one that contains both BubR1 and Mad1 and one containing only Mad1

99 (Figure 1D, left; phosphodomains that do not bind Mad1 cannot generate MCC). BubR1 interacts

100 with the Bub1 phosphodomain regardless of its phosphorylation state, whereas Mad1 interacts

101 only with the phosphorylated phosphodomain (Faesen et al., 2017; Ji et al., 2017). Therefore, the

102 latter interaction depends on the amount of Frb-mCherry-Mps $1^{500-817}$, i.e., the eSAC dosage. We

103 assume that the cellular concentration of the eSAC phosphodomain is higher than both BubR1

104 and Mad1 concentration (this is experimentally confirmed later). Consequently, the number of

105 signaling complexes saturates at high eSAC dosage (Figure S1A). Cdc20 recruitment to either

106 signaling complex is assumed to be independent of phosphorylation as well as BubR1 or Mad1

107 binding to the phosphodomain (not depicted).

108 The signaling complex containing Bub1, BubR1, Mad1, and Cdc20 facilitates the formation of 109 either the Mad2-Cdc20 complex or the MCC. Since the rate-limiting step in MCC assembly is the 110 formation of Mad2-Cdc20, we choose the same rate constant for the formation of MCC and Mad2-

111 Cdc20 (Faesen et al., 2017). We assume that a newly formed Mad2-Cdc20 at the phosphodomain 112 can combine with BubR1 to form the MCC with probability $p$, or escape to the cytoplasm with 113 probability $1-p$. Thus, the rates of MCC and Mad2-Cdc20 formation at the Bub1 phosphodomain 114 are given by:

MCC formation rate at Bub1 $1^{231-620}=p \cdot k_{\mathrm{asmcc} 2} \cdot[\operatorname{Mad} 2]_{\mathrm{I}} \cdot[\mathrm{Cdc} 20-\mathrm{Bub1} 1-\mathrm{BubR} 1]$ Mad2-Cdc20 formation rate at Bub1 ${ }^{231-620}$ or Bub1 $1^{444-620}$

118 where [Mad2], is the concentration of inactive (open) form of Mad2 in the cytoplasm and [Cdc20 119 -Bub1-BubR1] is the concentration of the complex between Bub1, BubR1, and Cdc20. This 120 simplified reaction scheme assumes MCC formation to be a one-step reaction. 
121 The important question is whether Bub1-BubR1 heterodimerization promotes MCC formation at

122 the Bub1 phosphodomain. If we assume that it does not contribute to MCC formation (or in the

123 case of Bub1 ${ }^{444-620}$, which lacks the BubR1 binding site), then only Mad2-Cdc20 will be produced

124 at the phosphodomain. This sub-complex must interact with BubR1 in the cytosol to complete

125 MCC formation. We calculate this rate as:

$$
\text { MCC formation rate in the cytoplsam }=k_{\text {asmcc1 }} \cdot[\text { BubR1 }]_{\mathrm{f}} \cdot[\operatorname{Mad} 2-C d c 20] \text {, }
$$

127 where $[\text { BubR1 }]_{\mathrm{f}}$ is the concentration of free BubR1 in the cytoplasm. To simulate the effect of 128 MCC generation by these two mechanisms on mitotic progression, we modified a previously 129 described model of the metaphase-to-anaphase transition (Chen et al., 2019; He et al., 2011) 130 (Methods, also see Figure S1B).

131 We first simulated the dose-response curve for Bub1 $1^{444-620}$ by assuming a suitable value for the $132 k_{\text {asmcc1 }}$ parameter (assumed protein concentrations and rate constants are listed in Table S1).

133 Next, we simulated dose-response data for Bub1 ${ }^{231-620}$. When we assumed that BubR1 binding to 134 the kinetochore does not affect the rate of MCC generation, i.e., $p=0$ in the above equations, 135 the simulation generated a weaker dose-response curve with a lower maximal response (dotted 136 blue line in Figure 1E). The response is weaker because BubR1 bound to Bub1 cannot participate 137 in cytosolic MCC assembly, and consequently, the rate of cytoplasmic MCC generation is 138 reduced. This result is inconsistent with the observed dose-response curves. The nominal choice 139 of $p=1$ and $k_{\text {asmcc2 }}=0.5 \mathrm{nM}^{-1} \mathrm{~min}^{-1}$ resulted in longer delays in anaphase onset - matching 140 the observed maximal response (Cyclin B dynamics shown in Figure S1C).

141 A comparison of the total MCC formation rates according to Eq. 1 and 2 shows that the cumulative 142 MCC formation rate is only modestly higher ( 12\%) when the BubR1 heterodimerization domain 143 is present in the phosphodomain (Figure S1D). However, this cumulative rate includes 144 contributions from both types of signaling complexes present in the cell. We wanted to assess the 145 magnitude of enhancement in MCC generation for each signaling complex. To roughly estimate 146 this local speed-up, we first observe that, in the case of a full Bub1 phosphodomain, the rate of 147 MCC formation is proportional to the concentration of Bub1-Cdc20-BubR1 (Figure S1E left). 148 Similarly, in the case of Bub1 ${ }^{444-620}$, the rate of MCC assembly in the cytoplasm is roughly 149 proportional to the Bub1-Cdc20 complex concentration (this complex must bind BubR1 in the 150 cytoplasm to complete MCC assembly, Figure S1E right). Therefore, upon taking the ratio of the 151 concentrations of these two complexes, we find that the rate of MCC assembly per 152 phosphodomain due to Bub1-Cdc20-BubR1 complex is $\sim 10$ times faster than the effective rate 
153

154

155

156

157

158

159

160

161

162

163

164

165

166

167

168

169

170

171

172

173

174

175

176

177

178

179

180

181

182

183

184

185

of MCC assembly due to Bub1-Cdc20 complex (note the difference in the scaling factors used to display the concentrations of the two complexes in Figure S1E).

\section{KI motifs suppress the signaling strength of the eSAC phosphodomain}

The KI motifs, so named because of the critical role that Lysine and iso-Leucine residues play in their activities, also recruit Bub1 and BubR1 to the human kinetochore (Figure 1A). The first KI motif $(K I 1)$ is thought to exclusively bind Bub1, whereas the second motif $(K I 2)$ is thought to bind BubR1 (Krenn et al., 2012). A prior study concluded that the KI motifs cooperate with the first MELT motif in KNL1 to strengthen SAC signaling (Krenn et al., 2014). However, it is unclear whether this is because Bub1 and BubR1 recruited by the KI motifs participate in MCC assembly or because they recruit regulatory enzymes (e.g., PLK1, PP2A) that promote SAC signaling (Nijenhuis et al., 2014). Therefore, to test whether Bub1 and BubR1 bound to the KI motifs directly promote MCC assembly and to delineate the roles of the two KI motifs, we developed another eSAC system. In this system, a fragment of Knl1 spanning the first MELT motif and the two KI motifs serves as the phosphodomain (Knl1 ${ }^{160-256}$, Figure 2A). Additionally, we created a variant phosphodomain wherein the two $\mathrm{KI}$ motifs are inactivated by mutating the residues identified as critical for their activity (Krenn et al., 2012). If Bub1 and BubR1 recruitment via the KI motifs enhances MELT motif activity, this will be apparent as increased signaling strength of the eSAC phosphodomain.

To establish the basal activity of the first MELT motif, we first studied the dependence of mitotic duration on the dosage of the eSAC signaling complex consisting of the eSAC phosphodomain with mutant, inactive $\mathrm{KI}$ motifs (M1-KI1*-KI2*-mNG-2xFkbp12, Figure 2A left). As before, the data was fit with a sigmoidal curve (Figure 2A, middle panel) (Chen et al., 2019). The maximal response for this phosphodomain was higher than the previously defined maximal response for the eSAC phosphodomain containing the $12^{\text {th }}$ MELT motif (211 versus 157 minutes with $\pm 95 \%$ confidence intervals of 169-357 and 152-163 respectively predicted by a 4-parameter sigmoidal fit to the binned data). This difference likely reflects the difference in the Bub1-Bub3 binding affinities of the first and twelfth MELT motif (Vleugel et al., 2015). Interestingly, the inclusion of active $\mathrm{KI}$ motifs in the phosphodomain significantly depressed its signaling strength: the eSAC concentration required for half-maximal response nearly doubled (EC50 = 9.7 and 19.4 a. u. respectively, Figure $2 \mathrm{~A}$ middle). Decrease in the apparent signaling strength of the phosphodomain was eventually compensated by high eSAC dosage, and therefore, the maximal response was similar in value (Figure $2 \mathrm{~A}$ middle and right). 
186 Thus, the inclusion of the KI motifs in the eSAC phosphodomain suppressed MCC production

187 mediated by the phosphorylated MELT motif within the same phosphodomain. Our eSAC-based

188 analyses of the Bub1 phosphodomain in the prior section provides a possible explanation for this

189 unexpected observation: if one assumes that the $\mathrm{KI}$ motifs interact with Bub1 and BubR1

190 independently of the MELT motif, and if the Bub1 and BubR1 molecules recruited by the KI motifs

191 do not participate in MCC formation mediated by the MELT motif, then the KI motifs will effectively

192 sequester Bub1 and BubR1 from MELT-motif mediated MCC assembly. This will suppress the

193 signaling activity of the eSAC.

195 Bub1 and BubR1 interactions with the KI motifs do not require Mps1-mediated 196 phosphorylation of KNL1; Bub1 interacts with both KI motifs

197 To delineate the activities of the KI motifs in the eSAC system, we constructed a new 198 phosphodomain by fusing an unstructured region of the Knl1 phosphodomain spanning three 199 previously characterized MELT motifs (KnI1 $\left.{ }^{881-1014}\right)$ to the C-terminus of the phosphodomain used 200 above (Knl1 ${ }^{160-256}$, see Figure 2B) (Chen et al., 2019; Vleugel et al., 2015). We also created mutant 201 versions of this phosphodomain wherein the $\mathrm{KI}$ motifs were rendered inactive either individually 202 or together using point mutations $\left(\mathrm{KI} 1^{*}-\mathrm{KI} 2, \mathrm{KI} 1-\mathrm{KI} 2^{*}\right.$, and $\mathrm{KI} 1^{*}-\mathrm{KI} 2^{*}$ with the asterisk denoting 203 loss of activity, Figure 2B) (Krenn et al., 2012). By incorporating multiple MELT motifs in the 204 phosphodomain, we wanted to test whether the KI motifs influence the ability of the four MELT 205 motifs in the phosphodomain to engage in synergistic signaling (Chen et al., 2019).

206 We first determined whether the two KI motifs interact with Bub1 and BubR1 exclusively, and 207 whether MELT motif phosphorylation is necessary for these interactions. We immunoprecipitated 208 the four, mNeonGreen-tagged eSAC phosphodomains from whole cell extracts of mitotic HeLa 209 cells in the absence of the Mps1 kinase domain using mNeonTrap beads and probed the 210 precipitates for Bub1 and BubR1 (Methods). When both KI motifs were active (KI1-KI2), Bub1 211 and BubR1 co-precipitated with the eSAC phosphodomain. When both $\mathrm{KI}$ motifs were inactive $212\left(\mathrm{KI} 1^{*}-\mathrm{KI} 2^{*}\right)$, Bub1 and BubR1 did not co-precipitate with the eSAC phosphodomain as expected 213 (Figure 2B). Surprisingly, when the first KI motif, which is expected to interact with Bub1 alone, 214 was inactive $\left(\mathrm{KI} 1^{*}-\mathrm{KI} 2\right)$, Bub1 still co-precipitated with the phosphodomain albeit at a lower level. 215 BubR1 coprecipitation was also reduced. This observation suggests that Bub1 competes with 216 BubR1 to bind to the second $\mathrm{KI}$ motif. Consistent with this inference, inactivation of second $\mathrm{KI}$ 217 motif $\left(\mathrm{KI} 1-\mathrm{KI} 2^{*}\right)$ made BubR1 undetectable in the precipitate and reduced the amount of Bub1. 
218 We obtained similar results from immunoprecipitation experiments involving eSAC

219 phosphodomains with only one MELT motif and the two KI motifs (Supplementary Figure S2A).

220 These results show that the first KI motif binds only Bub1, but the second motif can bind Bub1 or

221 BubR1. Furthermore, these interactions are not dependent on MELT motif phosphorylation.

223 Bub1 and BubR1 recruited by the KI motifs do not contribute to MCC assembly mediated 224 by the MELT motifs

225 Having established the biochemical activities of the $\mathrm{KI}$ motifs, we performed dose-response 226 analysis for the eSAC phosphodomain containing inactive KI motifs to define the baseline activity 227 of the four MELT motifs $\left(\mathrm{KI}^{*}{ }^{*}-\mathrm{KI} 2^{*}\right.$ in Figure 2B left, purple circles). The response elicited by the 228 eSAC phosphodomain with four MELT motifs to increasing eSAC concentration was non229 monotonic and characterized by an initial steep rise that is followed by a gradual decay in the 230 mitotic duration (residuals from a sigmoidal fit to the dose-response data points is shown in Figure 231 S2B, left). It should be noted that such non-monotonic response was not observed for a previously 232 characterized eSAC phosphodomain also containing four MELT motifs (numbers 11-14) (Chen et 233 al., 2019). The different behaviors of the two phosphodomains may be due to a higher affinity of 234 the $1^{\text {st }}$ MELT motif compared to the $11^{\text {th }}$ MELT motif for binding Bub3-Bub1 (Vleugel et al., 2015). 235 Interestingly, when both $\mathrm{KI}$ motifs were active, the maximal duration of mitosis was significantly 236 reduced ( $\sim 87$ minutes estimated by 4-parameter sigmoidal fit to the binned averages of the data, 237 see Figure 2C). Qualitatively similar results were obtained for an extended phosphodomain containing 7 MELT motifs and the two KI motifs (Figure S2C).

239 We next determined the dose-response dependence for two eSAC phosphodomains containing 240 four MELT motifs and only one active KI motif. When only the first KI motif was active (KI1-KI2*, 241 only Bub1 depleted, see Figure 2B), the dose-response data were monotonic with a slightly lower 242 maximal response (Figure $2 \mathrm{C}$ green circles and curve). When only the second $\mathrm{KI}$ motif was active $243\left(\mathrm{KI} 1^{*}-\mathrm{KI} 2\right.$, Bub1 and BubR1 depleted, Figure 2C), the maximal response was significantly 244 attenuated (blue circles and line in Figure 2C). Interestingly, the response to this eSAC was non245 monotonic: an initial overshoot was followed by decay to a lower maximal response (residuals 246 from the sigmoidal fit in Figure S2B, right). These results further show that Bub1 and BubR1 247 recruited by the two KI motifs do not directly contribute to MCC generation by the MELT motifs 248 within the same phosphodomain in the eSAC system (Figure 2C). The likely explanation for the 249 suppression of eSAC signaling activity is that Bub1 and BubR1 recruitment by the $\mathrm{KI}$ motifs 250 sequesters these proteins preventing them from participating in eSAC signaling. 
251 One simple explanation for the strong suppression of eSAC signaling activity by the second $\mathrm{KI}$

252 motif is that this motif sequesters BubR1 along with Bub1. Since BubR1 is an MCC component,

253 its sequestration will limit the maximal amount of $M C C\left(M C C_{\max }\right)$ that the eSAC can generate.

254 Although we could not quantify the amount of MCC produced by the eSAC, this amount and

$255 M C C_{\max }$ can both be compared qualitatively by studying the duration of mitotic delays. The eSAC

256 systems discussed here delay mitosis by at most 300 minutes. In contrast, RPE-1 cells treated

257 with high doses of nocodazole arrest for $~ 1500$ minutes on average (Collin et al., 2013; Dick and

258 Gerlich, 2013). The significantly smaller delay caused by eSAC signaling, when compared to the

259 delay induced by nocodazole treatment, suggests that the eSAC produces MCC levels smaller

260 than $M C C_{\max }$ (Dick and Gerlich, 2013). Therefore, a reduced $M C C_{\max }$ due to BubR1 sequestration

261 by the second $\mathrm{KI}$ motif alone cannot explain the weaker signaling activity.

\section{Numerical simulation of the dose-response data}

264 We returned to numerical simulations to understand the mechanistic underpinnings of the doseresponse data. The suppression of eSAC signaling activity by the $\mathrm{KI}$ motifs is in part because the eSAC phosphodomains are significantly more abundant than Bub1 and BubR1 (shown later). Nonetheless, the quantitative dose-response data provided us with an excellent opportunity to delineate the contribution of BubR1 recruitment at the MELT motifs to MCC formation. Bub1 and BubR1 sequestration will differentially affect the composition of the two signaling complexes: MELpT-Bub1 and MELpT-Bub1-BubR1 that the eSAC phosphodomains can assemble. If these

271 signaling complexes assemble the MCC at different rates, their effects on mitotic progression will

272 also be different (Dick and Gerlich, 2013). Using simulations, we wanted to understand the 273 mechanisms that give rise to the $\sim 1.75$-fold difference in the maximal mitotic delay generated by $274 \mathrm{KI} 1-\mathrm{KI} 2^{*}$ and $\mathrm{KI} 1^{*}-\mathrm{KI} 2$ (Figure $2 \mathrm{C}$ ).

275 To understand underlying mechanisms, it is necessary to consider: (a) the effects of the 276 differential sequestration of Bub1 and BubR1 by the two KI motifs on the concentrations of the 277 two signaling complexes, and (b) the rates at which these signaling complexes generate MCC. 278 Both types of signaling complexes will form on the eSAC phosphodomain, because we have 279 previously shown the eSAC phosphodomain recruits Bub1, BubR1, and Mad1 only in cells treated 280 with rapamycin (Chen et al., 2019). The stoichiometry of these two signaling complexes depends 281 on the interplay of two sets of parameters: (1) the stoichiometry of Bub1, BubR1, and the eSAC 282 phosphodomain (quantification presented after the simulations), and (2) the relative affinities of 
283 Bub1 and BubR1 for the two KI motifs, of Bub1 for phosphorylated MELT motifs, and of BubR1

284 for Bub1, which are known (Krenn et al., 2014; Vleugel et al., 2015; Zhang et al., 2016).

285 To simulate the activities of the KI motifs, we added two new elements to our previously described 286 model of the eSAC (Chen et al., 2019): the two KI motifs, and the factors Bub1 and BubR1 287 representing Bub1-Bub3 and BubR1-Bub3 respectively (Figure 3A). As before, all interactions are 288 described by mass action kinetics. In accord with our IP results (Figure 2), the model assumes 289 that the first $\mathrm{KI}$ motif $(\mathrm{KI} 1)$ interacts only with Bub1, whereas the second $\mathrm{KI}$ motif $(\mathrm{KI})$ can interact 290 with either Bub1 or BubR1. Based on prior observations, we also assume that only BubR1-Bub3 291 is recruited by the Bub1 bound to a phosphorylated MELT motif (Overlack et al., 2017; Overlack 292 et al., 2015). Based on in vitro data, the dissociation constant for Bub1 and BubR1 bound to the $293 \mathrm{KI}$ motifs was assumed to be 6 to 50 -fold higher relative to the dissociation constant for Bub1 294 bound to a phosphorylated MELT motif (Chen et al., 2019; Krenn et al., 2012; Overlack et al., 295 2015; Primorac et al., 2013) (rate constants tabulated in Table S2). We assume that the KI motifs 296 and phosphorylated MELT motifs recruit Bub1 and BubR1 independently. Finally, we also assume 297 that Bub1 and BubR1 bound to the KI motifs do not participate in the signaling events mediated 298 by the phosphorylated MELT motifs (Figure 3A). Three of the four MELT motifs used in the eSAC 299 phosphodomain were used in our previous study. Therefore, their affinities for Bub1 were similar 300 to the values used in the original simulation (Chen et al., 2019). The rate constants for the first, $30112^{\text {th }}$ and $14^{\text {th }}$ MELT motifs were set to reflect that they have a higher affinity for Bub1 compared 302 to the weaker $13^{\text {th }}$ MELT motif (Vleugel et al., 2015). The protein concentrations used are 303 tabulated in Table S2.

Effects of the differential activity of the KI motifs on the distribution of the signaling complexes formed by the eSAC phosphodomains

307 We first calculated the steady-state concentrations of MELpT-Bub1 and MELpT-Bub1-BubR1 assembled by $\mathrm{KI} 1-\mathrm{KI} 2^{*}$ and $\mathrm{KI} 1^{*}-\mathrm{KI}$. Bub1-BubR1 stoichiometry plays a critical role in determining these concentrations. If Bub1-BubR1 stoichiometry is high, e.g., 1:4, nearly every MELpT-Bub1 complex assembled by $\mathrm{KI} 1-\mathrm{KI} 2^{*}$ and $\mathrm{KI}{ }^{*}-\mathrm{KI} 2$ will recruit BubR1 to form MELpTBub1-BubR1 despite the differential depletion of Bub1 and BubR1 by the two phosphodomains

312 (converging dashed lines at high eSAC dosage in Figure 3B, right). Consequently, the 313 concentration of MELPT-Bub1 is negligible (dashed green and blue lines respectively near the $X$ 314 axis in Figure 3B, left). At high eSAC dosage, both $\mathrm{KI} 1-\mathrm{KI} 2^{*}$ and $\mathrm{KI}{ }^{*}-\mathrm{KI} 2$ form the same signaling 315 complex (MELpT-Bub1-BubR1). Because both phosphodomains form similar amounts of the 
same signaling complex, they will generate similar amounts of MCC, and therefore produce

317 mitotic delays of similar magnitude.

318 This picture changes when Bub1-BubR1 stoichiometry is around 1:1. In this case, KI1-KI2* and $319 \mathrm{KI}{ }^{*}-\mathrm{K} 12$ phosphodomains assemble different equilibrium concentrations of MELpT-Bub1 and 320 MELpT-Bub1-BubR1 especially at high eSAC dosage. BubR1 depletion by KI1*-KI2 lowers the 321 amount of MELpT-Bub1-BubR1 that it forms compared to $\mathrm{KI} 1-\mathrm{KI}{ }^{*}$ (Figure 3B, right, solid lines).

322 Conversely, the concentration of MELpT-Bub1 formed by $\mathrm{KI}{ }^{*}-\mathrm{KI} 2$ is now higher, because BubR1 323 availability is reduced (solid lines in Figure 3B, left). Figure 3C shows how Bub1-BubR1 324 stoichiometry affects MELpT-Bub1-BubR1 concentration (Figure 3C left, for the high and constant 325 eSAC dosage $=20$ ). These calculations show that if the $\mathrm{KI} 1-\mathrm{KI}{ }^{*}$ and $\mathrm{KI} 1^{*}-\mathrm{KI} 2$ phosphodomains 326 are to exhibit different dose-response characteristics, BubR1 (i.e., BubR1-Bub3) cannot be in too 327 much excess of Bub1 (i.e., Bub1-Bub3). Equal Bub1 and BubR1 abundance will produce the 328 maximal difference in the levels of signaling complexes assembled by $\mathrm{KI} 1-\mathrm{KI} 2^{*}$ and $\mathrm{KI} 1^{*}-\mathrm{KI} 2$ at 329 high eSAC dosage. This difference in the composition of the signaling complexes can provide the 330 basis for the differential activity of the $\mathrm{KI} 1-\mathrm{KI}{ }^{*}$ and $\mathrm{KI} 1{ }^{*}-\mathrm{KI} 2$ phosphodomains.

A higher rate of MCC generation by MELpT-Bub1-BubR1 compared to MELpT-Bub1 can explain the differential behavior of the two phosphodomains

Differences in the maximal concentrations of the MELPT-Bub1 and MELpT-Bub1-BubR1 will translate into different levels of MCC only if they generate MCC at different rates (the ratio of maximal mitotic duration achieved by $\mathrm{KI} 1-\mathrm{KI} 2^{*}$ and $\mathrm{KI} 1^{*}-\mathrm{KI} 2$ is indicated by the red horizontal line in Figure 3C). Specifically, MELpT-Bub1-BubR1 must generate MCC at a higher rate than MELpT-Bub1. To simulate MCC generation by the two signaling complexes, we used a simplified process of MCC generation wherein we do not explicitly include the dynamics of Mad2, Bub1 and BubR1. Instead, a single reaction between the signaling complex and free cytoplasmic Cdc20 generates MCC, with the forward rate constants $k_{M C C}$ for MELPT-Bub1 and $k_{M C C}^{\prime}$ for MELpT-Bub1BubR1 as the only parameters. This simplified, coarse-grained scheme is justified because, the rate of MCC generation at the phosphodomain and in the cytoplasm will be proportional to the concentration of the corresponding signaling complex (see Figure S1E). To simulate the effect of the MCC generated in this manner on mitotic progression, we suitably modified the model of metaphase-to-anaphase transition described by He et al. to reflect the simplified MCC generation scheme (Figure S3B) (Chen et al., 2019; He et al., 2011). 
To study how $k_{M C C}$ and $k_{M C C}^{\prime}$ affect the maximal mitotic duration, we fixed the eSAC dosage at a high and constant value, and then calculated the ratio of maximal mitotic duration produced by $\mathrm{KI} 1-\mathrm{KI} 2^{*}$ over that produced by $\mathrm{KI} 1^{*}-\mathrm{KI} 2$ as a function of Bub1-BubR1 stoichiometry and $k_{M C C}{ }^{\prime} k_{M C C}$ ratio (contour plot in Figure 3D). From this plot we see that the simulations of the ratio 352 of maximal mitotic durations for $\mathrm{KI} 1-\mathrm{KI} 2^{*}$ and $\mathrm{KI} 1^{*}-\mathrm{KI} 2$ is 1.4 (close to the observed ratio of 1.75), when (1) BubR1-Bub1 stoichiometry is 1, and (2) the signaling strength of the MELpTBub1-BubR1 signaling complex is at least 6-fold higher than the signaling strength of the MELpTBub1 signaling complex.

We used this framework to simulate the dose-response curves for all four phosphodomains. In this simulation, we assumed [Bub1]:[BubR1] $=1$ and $k_{M C C}: k_{M C C}^{\prime}=0.1$ (corresponding to a 10-fold increase in MCC generation at the ESAC phosphodomain relative to cytoplasmic MCC generation). With these nominal parameter values, dose-response curves generated by the model capture key characteristics of the dose-response data (Figure 3E). As before, the assumption of synergistic signaling is necessary to reproduce the non-monotonicity of the dose-response data for the phosphodomain containing four MELT motifs (Chen et al., 2019). Without it, the responses elicited by all phosphodomains become monotonic (Figure S3D). An interesting feature of the observed dose-response data for the $\mathrm{KI} 1^{*}-\mathrm{KI} 2$ phosphodomain is their non-monotonic nature (Figure 2E). Our simulations suggest that this difference arises due to Bub1 sequestration by the $\mathrm{KI}$ motif, which in turn suppresses synergistic signaling. Synergistic signaling by MELT motifs requires high occupancy of MELpT motifs by Bub1-BubR1. For this, the binding between MELpT and Bub1 is the necessary first step. In the $\mathrm{KI} 1^{*}-\mathrm{KI} 2 \mathrm{eSAC}$, Bub1 is not sequestered as strongly. This allows the assembly of multiple MELPT-Bub1-BubR1 complexes on the same phosphodomain only at low eSAC concentration. Resultant synergistic signaling becomes observable as a peak in the dose-response curve. In the case of the KI1-KI2* eSAC, such synergistic signaling is modulated due to Bub1 sequestration, and cannot be detected. We found that simply increasing the dissociation constant of KI1-Bub1 binding by a factor of two (thereby decreasing Bub1 sequestration) made the dose-response curve for KI1-KI2* eSAC visibly nonmonotonic (not shown).

\section{Stoichiometry of Bub1, BubR1, Mad1, and the eSAC phosphodomain in HeLa cells}

378 The simulations show that the stoichiometric ratios among Bub1, BubR1, and the eSAC 379 phosphodomain are critical determinants of the behavior of the eSAC systems. To experimentally 380 measure these ratios, we constructed genome-edited HeLa cell lines wherein mNeonGreen 
381 (abbreviated as $\mathrm{mNG}$ ) was fused to the N-terminus of Bub1 and BubR1, and to the C-terminus of

382 Mad1 (see Materials and Methods). In all three cases, we obtained clonal cell lines with partially

383 edited genomes: approximately half of the protein was labeled; the remaining protein fraction was

384 unlabeled (Figure 4A, also see Methods).

385 Using mNG fluorescence quantitation, we found that the abundance of the eSAC phosphodomain 386 containing two active $\mathrm{KI}$ motifs and four MELT motifs was 2-4-fold higher than the three SAC 387 proteins in mitotic cells (Figure 4B). The over-abundance of the eSAC phosphodomain relative to 388 Bub1, BubR1, and Mad1, and the constitutive activity of the two KI motifs together support our 389 assumption that the highly expressed eSAC phosphodomains sequester Bub1, BubR1 or both. 390 Moreover, BubR1 is 2-fold more abundant than both Bub1 and Mad1 (Figure 4C). It should be 391 noted that this abundance corresponds to the total BubR1 in a cell including free BubR1-Bub3 as 392 well as BubR1-Bub3 incorporated into MCC (Overlack et al., 2017). The partitioning of BubR1 393 between the BubR1-Bub3 complex and the MCC remains unknown. Therefore, we conclude that 394 the concentration of BubR1-Bub3 available for SAC signaling is less than 2-fold higher than that 395 of Bub1-Bub3. This is consistent with the prediction of our model that the BubR1:Bub1 396 stoichiometry must be near $1: 1$ for the observed dose-response data for the $\mathrm{KI} 1-\mathrm{KI} 2^{*}$ and $\mathrm{KI} 1^{*}$ $397 \mathrm{KI} 2$ phosphodomains.

\section{Recruitment of BubR1 by BuB1 per se contributes to the signaling strength of the SAC}

400 Observations from the eSAC systems and our numerical simulations forced us to re-examine the 401 role of Bub1-BubR1 heterodimerization in kinetochore-based SAC signaling. Previous studies 402 using the knock-down and rescue approach found that the disruption of Bub1-BubR1 403 heterodimerization increases the SAC strength in nocodazole-treated cells, increasing the 404 duration of mitotic arrest rather than decreasing it (Krenn et al., 2014; Zhang et al., 2016). 405 However, we noted three factors that may have influenced this result. First, the deletion of different segments of BubR1 in the two studies may have resulted in different levels of residual BubR1 at 407 signaling kinetochores. The second factor arises from the loss of the SAC-antagonizing activity 408 of PP2A due to the disruption of BubR1 recruitment to the kinetochore. The kinetochore 409 attachment and regulatory domain (KARD) of BubR1 was either left intact or mutated to eliminate 410 the binding of the PP2A-recruiting subunit B56a, but not necessarily its other isoforms. Most 411 importantly, the mutant BubR1 may have been overexpressed in these experiments. Higher levels 412 of BubR1 in the cytosol will proportionally increase the rate of cytosolic MCC formation, and the 413 increased rate may mask impaired MCC assembly within kinetochores. 
414 To account for these three factors, we designed and performed new BubR1 knock-down/rescue

415 experiments. We knocked down endogenous BubR1 in HeLa-A12 using RNA interference and

416 rescued these cells with $m$ NG-BubR $1^{\Delta 665-682}$ or $m N G-B u b R 1^{\Delta 432-484, \Delta 665-682}$. Both lack the KARD to

417 interact with PP2A-B56 ( $\triangle K A R D)$, while mNG-BubR1 ${ }^{\Delta 432-484, ~} \Delta 665-682$ cannot heterodimerize with

418 Bub1 $(\triangle H D, \triangle K A R D)$. Therefore, mNG-BubR1 ${ }^{\triangle 432-484, \Delta 665-682}$ has nearly undetectable localization

419 at unattached kinetochores (Figure 5A). In these cells, we induced mitotic arrest using nocodazole

420 treatment following the previous studies. Importantly, we used the genome-edited $m N G-B U B R 1$

421 HeLa-A12 cells treated with control siRNA and imaged under identical conditions to establish the

422 physiological BubR1expression level. In our analysis of cells expressing the mutant, mNG-tagged

423 BubR1, we only considered those cells exhibiting mNG intensity that is 0.5-2-times the average

424 mNG intensity of mitotic mNG-BubR1 HeLa-A12 cells (because only around half of total BubR1

425 is tagged in the CRISPR-Cas9-edited cell line, Figure 5B, left).

426 We found that in BubR1 knock-down cells rescued with BubR1 lacking the KARD domain arrested

427 for a significantly longer duration as compared to control cells. This is likely due to the loss of

428 PP2A activity from the kinetochores (Saurin et al., 2011). Importantly, cells rescued with BubR1

429 lacking both the KARD domain and the Bub1 heterodimerization domain arrested for a 430 significantly shorter amount of time in comparison (Figure 5B, right). Thus, the recruitment of 431 BubR1 per se contributes to the efficient assembly of MCC.

\section{Discussion}

434 Unattached kinetochores are poised to assemble the MCC by recruiting its component subunits: 435 BubR1-Bub3, Mad2, and Cdc20. Therefore, it was surprising that the disruption of BubR1 436 recruitment to the kinetochore was found to be unimportant for SAC signaling in nocodazole437 treated cells (Overlack et al., 2015; Zhang et al., 2016). We used the eSAC system to isolate a 438 subset of the biochemical reactions in the MCC generation cascade from the kinetochore and 439 analyzed them in the physiological context. These analyses show that Bub1-BubR1 440 heterodimerization significantly enhances the rate of Bub1-mediated MCC assembly. This 441 enhancement may be simply due to enrichment of BubR1 at the site of formation of Mad2-Cdc20

442 (Lara-Gonzalez et al., 2021; Piano et al., 2021), although more complicated mechanisms may 443 also be at work. 
444 We also find that Bub1 and BubR1 recruitment via the KI motifs does not contribute to Bub1-

445 mediated MCC assembly. It is important to note that the eSAC signaling complexes contain only

446 one $\mathrm{Knl} 1$ phosphodomain in contrast to the spatially clustered organization of many Knl1

447 phosphodomains within the kinetochore. Therefore, it remains possible that KI motifs directly or

448 indirectly prime SAC signaling by constitutively recruiting Bub1 and BubR1 (Jia et al., 2016; Krenn

449 et al., 2014). Nonetheless, because of the higher affinity of phosphorylated MELT motifs for Bub1-

450 Bub3 and their superior numbers ( 19 MELT motifs versus $1 \mathrm{KI}$ motif each per Knl1), the MELT

451 motifs will take over Bub1 and BubR1 recruitment once Mps1 becomes fully active within

452 unattached kinetochores. KI motif activity is unlikely to contribute significantly under this condition.

453 We also constructed a numerical model to simulate the dose-response data using experimental 454 data wherever possible and assuming plausible parameter values when data were unavailable.

455 This simple model based on differential sequestration of Bub1 and BubR1 can simulate key 456 features of the dose-response data. The simulations support the following main conclusions. First, 457 Bub1-BubR1 heterodimerization significantly elevates the rate of Bub1-mediated MCC 458 generation. To simulate the observed dose-response data, the rate of MCC assembly due to 459 Bub1-bound BubR1 was assumed to be 10-fold higher when compared to cytoplasmic MCC 460 assembly in our model. This enhanced MCC generation rate is reasonable given that the local 461 concentration of BubR1 will increase by orders of magnitude upon its binding to Bub1, the site 462 where Mad2-Cdc20 is formed. Second, our model further supports the observation that Bub1 and 463 BubR1 recruited by the KI motifs do not contribute to MCC generation. Third, the stoichiometry of 464 Bub1 and BubR1 emerges as a key parameter influencing how the MCC generation output of 465 each kinetochore will change as a function of the number of signaling kinetochores in the cell. 466 These simulations thus establish a useful platform for future studies of SAC signaling.

467 Human kinetochores also use the RZZ pathway to promote MCC assembly, and this pathway can 468 compensate for a partial loss of Bub1 from the kinetochore (Qian et al., 2017; Silio et al., 2015; 469 Zhang et al., 2015). Nevertheless, MELT motif and Bub1-mediated MCC assembly is likely to be 470 critical over the course of a normal cell division. Bub1-mediated recruitment of BubR1 to the 471 kinetochore is especially important because this will significantly enhance the rate of MCC 472 generation within the kinetochore, unlike the RZZ pathway, which likely generates only Mad2473 Cdc20 and relies on cytosolic assembly of the MCC. Our study does not clarify the role of the KI 474 motifs. The possibilities include the 'priming' of SAC signaling in the early stages of mitosis by 475 enhancing local Bub1 and BubR1 concentration and the recruitment of regulatory factors via Bub1 476 and BubR1 for kinetochore functions unrelated to SAC signaling. 
479 We would like to thank Dr. Stephen S. Taylor for the generous gift of antibodies, Dr. Eugene 480 Makeyev for the HeLa acceptor cell line, and Dr. Michael Lampson for plasmids used in this study.

481 We also thank members of the Joglekar lab for stimulating discussions. Use of the Incucyte 482 system was made possible by a generous gift from the Richard Tam Foundation to Prof. Sue 483 O'Shea (Cell \& Developmental Biology, University of Michigan Medical School).

486 The authors declare that no competing financial interests exist. 


\section{Materials and Methods}

\section{Plasmid construction}

489 The plasmids used for the stable cell lines were based on plasmids that have been described 490 previously (Chen et al., 2019). Briefly, the phosphodomain was integrated either Notl or Ascl and 491 Xhol restriction sites to create constitutively expressed phosphodomain-mNeonGreen-2xFkbp12. 492 A Mps1 $1^{500-857}$ fragment corresponding to the Mps1 kinase domain was integrated into the Fsel 493 and $B g / l l$ restriction sites to create conditionally expressed Frb-mCherry-Mps $1^{500-857}$.

494 eSAC phosphodomains spanning the $1^{\text {st }}$ MELT motif (M1) and the two KI motifs were created by 495 fusing $\mathrm{Knl}^{160-256}$ to mNeonGreen-2xFkbp12. eSAC phosphodomains containing four MELT 496 motifs and the two KI motifs were created by fusing $\mathrm{Knl}^{160-256}$ to $\mathrm{Knl1}$ fragment $\mathrm{Knl} 1^{881-1014}$ 497 spanning MELT motifs 12-14, which has been characterized previously (Vleugel et al., 2015). The 498 activity of the first $\mathrm{KI}$ motif was disrupted by changing its amino acid sequence from 499 'KIDTTSFLANLK' to 'KADAASALANLK' (KI1*). Similarly, the activity of the second KI motif was 500 disrupted by mutating its amino acid sequence from 'KIDFNDFIKRLK' to 'KIDFNDAIKALK' (KI2*) 501 following (Krenn et al., 2012).

502 DNA repair templates used for CRISPR/Cas9 mediated genome editing were constructed via 503 DNA assembly using the NEB HiFi DNA assembly kit as per manufacturer's instructions. 504 Successfully edited alleles encode mNeonGreen-tagged SAC proteins that separate the 505 corresponding wildtype protein and the fluorescent protein mNeonGreen by a short flexible linker 506 (mNG-BUBR1 and mNG-BUB1: GSGGSG; MAD1-mNG: GGAGGSGG). The sequences of all 507 homology-directed repair template plasmids and Cre-Lox recombination-mediated cassette 508 exchange plasmids are available upon request.

Tissue culture and cell line construction for eSAC analyses

511 Henrietta Lacks (HeLa) cells were grown in DMEM media supplemented with 10\% FBS, 1\% 512 Pen/Strep, 1x-Glutamax, and 25 mM HEPES under standard tissue culture conditions $\left(37^{\circ} \mathrm{C}\right.$ and

$5135 \%$ CO2). Stable cell lines expressing the two eSAC components were generated by integrating 514 a bi-cistronic eSAC plasmid at engineered lox sites in the HeLa genome according to the protocol 515 described in (Khandelia et al., 2011). Upon transfection, DMEM media supplemented with $1 \mu \mathrm{g} / \mathrm{ml}$ 516 Puromycin was used to select transformed cells, and all the colonies were pooled to culture the 517 transformed cells used in the experiments.

518 To conduct dose-response analysis, each eSAC cell line was plated $\sim 40-48$ hours prior to the 519 start of the experiment in DMEM media without Puromycin. Doxycycline was added at the time of 520 plating to induce the expression of Frb-mCherry-Mps1. Prior to imaging, the cells were washed 
521 with PBS. Fluorobrite media with 10\% FBS, 1\% Pen/Strep with or without Rapamycin were added

522 to each well.

523

\section{Genome editing HeLa cells using CRISPR/Cas9}

525 The guide RNAs (gRNAs) for in situ BUBR1 and BUB1 N-terminal mNeonGreen-tagging were 5'-

526 CAGGAUGGCGGCGGUGAAGA-3' and 5'-GGUUCAGGUUUGGCCGCUGC-3', respectively.

527 The gRNA for in situ MAD1 C-terminal mNeonGreen-tagging was 5'-

528 CAGACCGUGGCGUAGCCUGC-3'. Single guide RNAs (sgRNAs) were synthesized using the

529 EnGen sgRNA Synthesis Kit (for the Streptococcus pyogenes-originated Cas9, New England

530 Biolabs). The SpCas9-sgRNA ribonucleoprotein (RNP) complex was assembled at room

531 temperature in a buffer consisting of $20 \mathrm{mM}$ of HEPES-KOH (pH 7.5), $150 \mathrm{mM}$ of KCl, $1 \mathrm{mM}$ of

$532 \mathrm{MgCl}_{2}$, ten percent (by volume) of glycerol, and $1 \mathrm{mM}$ of DTT using 100 pmol of SpCas9- 2×NLS

533 (the QB3 MacroLab) and 120 pmol of sgRNA. The RNP complex and $1.5 \mu \mathrm{g}$ of a linearized

534 homology-directed repair template plasmid were transfected into $2 \times 10^{5}-5 \times 10^{5}$ nocodazole-

535 arrested mitotic HeLa A12 cells using a Nucleofector and the associated Cell Line Kit R (Lonza)

536 following manufacturer's instructions. After five weeks, green fluorescence-positive mitotic cells

537 (arrested by $330 \mathrm{nM}$ of nocodazole for 16 hours) were sorted directly into 96-well plates at 1

538 cell/well. Healthy colonies were subject to further validation by genotyping and sequencing, as

539 well as immunoblotting.

540 For genotyping, HeLa-A12 genomic DNAs were purified using the Wizard SV Genomic DNA

541 Purification System (Promega). Genotyping primers (BUBR1 forward primer 5'-

542 CCTGGTCACATCTGAGCTAT-3', BUBR1 reverse primer 5'-CTCAGTGAGACTCCAGTGTT-3',

543 BUB1 forward primer 5'-CCCTCTACATGAAGGCGCTA-3', BUB1 reverse primer 5'-

544 GCTCGCCCAAGGTAAACATT-3', MAD1 forward primer 5'-GGACTTTTCAGGGACGTGGT-3',

545 and MAD1 reverse primer 5'-GAGTTGGGAGGAGGGGACTC-3') were designed to bind outside

546 of homology arms to avoid false-positive colonies from integration of the homology-directed repair

547 template plasmid to an off-target genomic locus.

548

\section{Drug and RNAi treatments}

550 To induce the expression of the mCherry-Frb-Mps1 kinase domain doxycycline was added to a

551 final concentration of $2 \mathrm{ug} / \mathrm{ml}$ (stock concentration $2 \mathrm{mg} / \mathrm{ml}$ in DMSO) $\sim 48$ hours prior to the start

552 of the experiment. Prior to prior to the start of each experiment, Rapamycin was added $\sim 1$ hour

553 of the experiment to a final concentration of $500 \mathrm{nM}$ (stock concentration $500 \mu \mathrm{M}$ in DMSO) to

554 induce the dimerization of the eSAC kinase domain with the eSAC phosphodomain. Nocodazole 
555 was added to the final concentration of $330 \mathrm{nM}$ (stock concentration $330 \mu \mathrm{M}$ in DMSO). The

556 cocktail of siRNA against five different B56 isoforms was added to a final concentration of $40 \mathrm{nM}$

557 (stock concentration $10 \mu \mathrm{M}$ ). The siRNA sequences were obtained from ref. (Nijenhuis et al.,

558 2014). Cell cycle synchronization was in G1/S was achieved by treating cells with $2.5 \mathrm{mM}$

559 thymidine (from a $100 \mathrm{mM}$ stock in PBS) for 16-18 hours. Cells were washed with DMEM for

560 release from the $\mathrm{G} 1 / \mathrm{S}$ arrest.

561

562 Immunoprecipitation

563 HeLa A12 cells constitutively expressing either MELT1-KI or MELT1-KI-M3 were 564 synchronized at G1/S by $2.5 \mathrm{mM}$ of thymidine. 9 hours after being released from the 565 (double) thymidine block, cells were synchronized in metaphase using $10 \mu \mathrm{M}$ MG132. 566 After another 1.5 hours, cells were scraped off the plate, washed once with PBS, pelleted, 567 snap-frozen, and stored at $-80^{\circ} \mathrm{C}$. Cells were thawed, resuspended in the complete lysis 568 buffer (75 mM HEPES-HCl (pH 7.5), 150 mM KCl, 1.5 mM EGTA, 1.5 mM MgCl2, 10\% 569 (by volume) glycerol, and 0.075\% (by volume) Nonidet P-40 (AmericanBio); immediately 570 before use one cOmplete protease inhibitor cocktail tablet (EDTA-free, Roche 571 Diagnostics) and a phosphatase inhibitor cocktail (1 mM Na4P2O7, $0.1 \mathrm{mM} \mathrm{Na3VO4,} 5$ $572 \mathrm{mM} \mathrm{NaF}$, and $2 \mathrm{mM}$ sodium $\beta$-glycerophosphate) were added), and lysed for 1 hour at 4 $573{ }^{\circ} \mathrm{C}$ while rotating. Cell lysates were then centrifuged at $18,000 \mathrm{~g}$ for $25 \mathrm{~min}$ at $4{ }^{\circ} \mathrm{C}$. The 574 supernatant was subsequently cleared by control agarose beads for 1.5 hours at $4{ }^{\circ} \mathrm{C}$ to 575 reduce non-specific binding. Cleared supernatant was then mixed with mNeonGreen576 Trap agarose beads (ChromoTek) and rotated for 1.5 hours at $4{ }^{\circ} \mathrm{C}$. Beads were washed 5774 times using the lysis buffer. Finally, 2xLaemmli buffer (Bio-Rad Laboratories) 578 supplemented with $\beta$-mercaptoethanol was added to the beads. Samples were boiled in 579 a water bath for 10 min before subjecting to SDS-PAGE.

580 The following antibodies and working dilutions were used in immunoblotting:

581 SB1.3 antibody against BUB1 (Taylor et al., 2001), 1:500 or 1:1000, sheep polyclonal; 582 BUB3 antibody (Sigma B7811), 1:500, rabbit polyclonal; FKBP12 antibody (Abcam 583 ab2918), 1:2000, rabbit polyclonal; BUBR1 antibody (Bethyl A300-995A), 1:1000, rabbit 584 polyclonal. 


\section{Long term live-cell imaging of HeLa cells}

588 Imaging was conducted over a period of 24 hours as described in detail previously (Chen et al., 589 2019). We used either the Incucyte Zoom live cell imaging system (Sartorus Inc.) or the 590 ImageXpress Nano live cell imaging system (Molecular Devices Inc), both equipped with a 20x 591 Phase objective. To image cells on the Incucyte system, cells were plated in 12-well plastic tissue 592 culture plates, whereas they were plated in 24-well plate glass-bottom dishes (Corning) for the 593 ImageExpress Nano system. At each position, one phase, GFP, and mCherry image was 594 acquired every 10 minutes. The exposure times for mCherry and GFP images were adjusted to 595 minimize photobleaching while enabling accurate determination of intensity values. It should be noted that the excitation sources, optics, and detector on the ImageXpress Nano and the Incucyte 597 microscope are entirely different. Therefore, the mCherry intensity values across different experiments are not directly comparable.

\section{Image analysis}

601 Prior to intensity quantification, acquired images were pre-processed using functions from the

602 'Image Processing Toolbox' provided with Matlab as follows. First, the phase image sequence 603 was registered to remove any movement of the field of view between adjacent time point, and at 604 each time point, the same transform was applied to the GFP and mCherry images to register 605 them. Additionally, image intensity from a blank, unseeded well was used to for background 606 correction of the fluorescence channels. Next, GFP and mCherry fluorescence signals were 607 quantified using a custom graphical user interface (GUI) written in Matlab as described previously. 608 Briefly, this interface uses cross-correlation of each phase image with a circular kernel to identify 609 cells with circular shapes close to the diameter of the circular kernel. The centroids of these 610 shapes were then linked along the time axis. These images were presented to the user via the 611 GUI to: (a) discard false positive, non-mitotic cells or debris and (b) to visually correct the time of 612 entry into or exit from mitosis. The GUI then calculated the GFP and mCherry signals per cell as 613 the average fluorescence intensity

614 In all the dose-response assays discussed in this study, the phosphodomain is highly, and 615 constitutively, expressed in all cells, whereas the kinase domain is expressed conditionally by an 616 inducible promoter. Consequently, the amount of the kinase domain expressed varies from cell 617 to cell, and it is lower than the amount of the phosphodomain in most cells. Because of this design 618 of the eSAC system, the dosage of the dimerized signaling complex in a cell can be inferred from 619 the amount of Frb-mCherry-Mps $1^{500-817}$ in that cell. Therefore, we defined the dosage of the eSAC 620 signaling complex by quantifying the average mCherry fluorescence within a cell, and the 
621 response as the duration of mitosis (the amount of time that the cell spends with a spherical

622 morphology that is characteristic of mitotic HeLa cells).

623

624

625

626

627

628

629

630

631

632

633

634

635

636

637

638

639

640

641

642

643

644

645

646

647

648

649

650

651

652

\section{Mathematical modeling}

\section{Modeling of dose-response curve for eSAC systems with Bub1 phosphodomains}

We constructed this model to understand the mechanisms underlying delayed mitotic exit produced by the eSAC consisting of Bub1 $1^{221-620}$ and Bub1 ${ }^{441-620}$. In the equations below, we refer to these phosphodomains simply as 'Bub1'. The eSAC activator complex is formed by the dimerization of Bub1 with the kinase domain of Mps1. Upon phosphorylation by Mps1, Bub1 can bind Mad1/2. The concentrations of different species of the Bub1 phosphodomain are related by the equation:

$$
[\text { Bub1 }]_{\mathrm{T}}=[\text { Bub1-Mps1-Mad1/2] }+[\text { Bub1-Mps1] }+[\text { Bub1] }
$$

Assuming reversible binding between phosphorylated Bub1 phosphodomain and Mad1/2 complex, we determined the concentration of eSAC activator complex, [Bub1-Mps1-Mad1/2] - it saturates due to the finite concentration of Mad1-Mad2 complex (set at $100 \mathrm{nM}$, (Fig. S1A). Note, that the phosphodomain can produce MCC only if it recruits Mad1/2. Bub1-Mps1 and Bub1 can both bind BubR1 and Cdc20, but they do not participate in SAC signaling.

We modified a model of metaphase-to-anaphase transition by He et al. (He et al., 2011) as shown in Figure S1B to capture key proteins and steps in the process of Bub1-mediated MCC formation and to simulate the effect of MCC on mitotic exit. In the He model, Cyclin B ('CycB') is synthesized at a constant rate and degraded by APC-Cdc20-dependent ubiquitination (denoted simply as Cdc20). The abundance of $\mathrm{CycB}$ determines the activity of CDK1-CycB complexes. The activity of the eSAC complex depends positively on CDK1-CycB activity and negatively on PP2-B55, a counter-acting protein phosphatase (CAPP) (Bouchoux and Uhlmann, 2011; Sullivan et al., 2004). This is a reasonable assumption, because recent data reveals that Bub1 must be phosphorylated by both CDK1-CycB and Mps1 before it can bind Mad1 (Ji et al., 2017). The MCC undergoes spontaneous dissociation, releasing BubR1, Cdc20 and inactive/open Mad2. Furthermore, active APC-Cdc20 promotes the inactivation of closed/active Mad2 in MCC; this positive feedback of active Cdc20 on its own release from the MCC accelerates the activation of APC-Cdc20 during the transition into anaphase (Chen et al., 2019; He et al., 2011).

The molecular species, chemical reactions, parameters and constraints involved in the model are presented in an Excel file and Table S1 (Supporting Information). A MATLAB script was used to 
653

654

655

656

657

658

659

660

661

662

663

664

665

666

667

668

669

670

671

672

673

674

675

676

677

678

679

680

681

682

683

684

685

686

read this file and produce a file with Ordinary Differential Equations (ODEs) describing the rate of change of all molecular species. We numerically integrated the ODEs to calculate the time evolution of $[\mathrm{CycB}]$ and the other molecular species. For the initial conditions of species involving BubR1 and Cdc20 bound to Bub1 phosphodomain (both signaling and non-signaling) we used the equilibrium concentrations. These concentrations depended on the concentration of eSAC activator complex and were calculated at the start of each simulation. The initial concentrations of the following species were kept constant: $[\mathrm{CycB}]=45 \mathrm{nM}$, [Mad2-Cdc20] $=0 \mathrm{nM},[\mathrm{MCC}]=25$ $\mathrm{nM},[\mathrm{CAPP}]=5 \mathrm{nM}$. In our model the system temporally evolves towards a steady state corresponding to anaphase (low [CycB] and high [Cdc20]). We assume that a cell exits mitosis when [CycB] drops below $1 \mathrm{nM}$ (Fig. S1E). The choice of initial conditions does not qualitatively affect the outcome of the model. Furthermore, the main result of this analysis - Bub1-BubR1 produces MCC at a higher rate than Bub1 - is robust, even though many different combinations of parameters produce similar looking dose-response curves. Experimental measurements of some of these parameters will be needed to constrain the model further.

\section{Modeling of dose-response curve for ESAC with MELT and KI motifs}

We constructed this model to simulate the observed dose-response data for the different eSAC systems involving MELT and KI motifs. In these simulations, the phosphodomain consists of four MELT motifs and either active or inactive KI motifs. All four MELT motifs in contact with the Mps1 kinase domain are assumed to be phosphorylated, whereupon they can recruit Bub1 (representing Bub1-Bub3 in this model. Upon binding to the MELT motif, Bub1 recruits BubR1 (representing BubR1-Bub3). The KI1 motif can bind to Bub1, whereas the KI2 motif can bind to either Bub1 or BubR1 with characteristic binding affinities. The KI-bound Bub1 and BubR1 do not participate in SAC signaling. Therefore, the KI motifs act like sinks that reduce Bub1 and BubR1 concentration. Interaction of the MELT motifs with Bub1 and BubR1 produces two types of signaling complexes: MELpT-Bub1 or MELpT-Bub1-BubR1. For simplicity, we did not explicitly model in detail how these signaling complexes catalyze MCC formation. Instead, we assume that the MELpT-Bub1 and MELpT-Bub1-BubR1 complexes catalyze the assembly of MCC at the apparent rates constants $k_{M C C}$ and $k_{M C C}^{\prime}$. A schematic diagram of the molecular mechanism underlying this model is displayed in Figure S3A. The model simulates dose-response data in two stages.

1. eSAC signaling cascade. We first calculate the steady-state concentrations of SAC signaling proteins (Bub1 and BubR1) bound to the phosphorylated MELT motifs and KI motifs of an 
eSAC phosphodomain. Using the steady-state concentrations of MELpT-Bub1 and MELpTBub1-BubR1, we calculate the rates of generation of MCC by the two phosphodomains, assuming that these rates is proportional their steady-state concentrations.

2. Mitotic delay. In the second stage of the model, we simulate the duration of mitosis, according to the overall reaction scheme developed by $\mathrm{He}$ et al. and discussed in the previous section.

\section{Stage 1: Simulation of SAC protein recruitment by the eSAC phosphodomains}

We assume that MELT and KI motifs in the eSAC phosphodomain interact with SAC proteins independently. We assigned the same rate of binding of the 'Bub1' protein to each MELT motif ( $k f$ in Table S2), but assigned a low unbinding rate ( $k r$, Table S2) for the strong MELT motifs (MELT 1, 12 and 14), and a higher unbinding rate for the weak MELT motif (MELT 13) following previous studies (Chen et al., 2019; Vleugel et al., 2015). We also chose the dissociation constant for KI1-Bub1 binding to be equal to the dissociation constant for the KI2-BubR1 binding.

Each phosphorylated MELT motif can be in one of three possible states: MELpT (unbound MELT), MELpT-Bub1 (MELT bound by Bub1), and MELpT-Bub1-BubR1 (MELT bound by Bub1 and BubR1). Since there are four MELT motifs in each eSAC activator complex, the number of possible states for the phosphomimic become $3^{4}=81$. Similarly, each $\mathrm{KI}$ motif of the phosphodomain can be in two states: bound or unbound. The time evolution of concentrations of different Bub1 and BubR1 bound states of eSAC activator complex is given by:

$$
\dot{\mathbf{X}}=\mathbf{A X}
$$

where $\mathbf{X}=\left\{x_{1}, x_{1}, \ldots, x_{N}\right\}$ is a vector of concentrations of the $N=81$ different Bub1 and BubR1 binding states of the eSAC, and $\mathbf{A}$ is the rate matrix. The binding of Bub1 and BubR1 to KI motifs is described by the set of equations:

$$
\begin{gathered}
\dot{y}_{1}=\operatorname{kfbub} \cdot[\mathrm{Bub} 1]_{\mathrm{f}} \cdot y_{0}-\operatorname{krbub} \cdot y_{1}-\operatorname{kfbub} \cdot[\mathrm{Bub} 1]_{\mathrm{f}} \cdot y_{1}-\operatorname{kfbubr} \cdot[\mathrm{BubR} 1]_{\mathrm{f}} \cdot y_{1} \\
+\operatorname{krbubr} \cdot y_{4}+\operatorname{krbub}^{\prime} \cdot y_{5}
\end{gathered}
$$

$$
\dot{y}_{2}=\mathrm{kfbub} \cdot[\mathrm{Bub} 1]_{\mathrm{f}} \cdot y_{0}-\mathrm{krbub}^{\prime} \cdot y_{2}-\mathrm{kfbub} \cdot[\mathrm{Bub} 1]_{\mathrm{f}} \cdot y_{2}+\mathrm{krbub}^{\prime} \cdot y_{5}
$$

$$
\dot{y}_{3}=\operatorname{kfbubr} \cdot[\mathrm{BubR} 1]_{\mathrm{f}} \cdot y_{0}-\operatorname{krbubr} \cdot y_{3}-\mathrm{kfbub} \cdot[\mathrm{Bub} 1]_{\mathrm{f}} \cdot y_{3}+\mathrm{krbubr} \cdot y_{4}
$$

$$
\dot{y}_{4}=\operatorname{kfbubr} \cdot[\mathrm{BubR} 1]_{\mathrm{f}} \cdot y_{1}-\operatorname{krbubr} \cdot y_{3}-\operatorname{krbub} \cdot y_{3}-\operatorname{krbubr} \cdot y_{4}
$$

$$
\dot{y}_{5}=\mathrm{kfbub}^{\prime} \cdot[\mathrm{Bub} 1]_{\mathrm{f}} \cdot y_{1}+\mathrm{kfbub} \cdot[\mathrm{Bub} 1]_{\mathrm{f}} \cdot y_{2}-\mathrm{krbub} \cdot y_{5}-\mathrm{krbub}^{\prime} \cdot y_{5},
$$

where, $y_{0}$ is the concentration of free $\mathrm{KI}$ motifs (both $\mathrm{KI}$ motifs unbound), 


$$
y_{1}=[\mathrm{KI} 1-\mathrm{Bub} 1]
$$

$$
y_{2}=[\mathrm{KI} 2-\mathrm{Bub} 1]
$$

$$
y_{3}=[\mathrm{KI} 2-\mathrm{BubR} 1] \text {, }
$$

$$
y_{4}=[\mathrm{KI} 1-\mathrm{Bub} 1, \mathrm{KI} 2-\mathrm{BubR} 1] \text {, }
$$

$$
y_{5}=[\mathrm{KI} 1-\mathrm{Bub} 1, \mathrm{KI} 2-\mathrm{Bub} 1] .
$$

$722[\mathrm{Bub} 1]_{\mathrm{f}}$ and $[\mathrm{BubR} 1]_{\mathrm{f}}$ are the concentrations of free Bub1 and BubR1 in the cytoplasm, 723 respectively. The parameters kfbub and $\mathrm{kfbub}^{\prime}$ are the rate constants for Bub1 binding to the first 724 and the second $\mathrm{KI}$ motif respectively (krbub and krbub' are the corresponding unbinding rates), 725 and kfbubr and krbubr are the binding (unbinding) rates between BubR1 and KI2.

726 The concentrations satisfy the constraints:

$$
[\mathrm{eSAC}]=\sum_{i=1}^{N} x_{i}
$$

$$
[\mathrm{Bub} 1]_{\mathrm{T}}=[\mathrm{Bub} 1]_{\mathrm{f}}+y_{1}+y_{2}+y_{4}+2 y_{5}+\sum_{i=1}^{N} n_{i} \cdot x_{i}
$$

$$
[\text { BubR } 1]_{\mathrm{T}}=[\text { BubR1 }]_{\mathrm{f}}+y_{3}+y_{4}+\sum_{i=1}^{N} m_{i} \cdot x_{i}
$$

Here, $x_{i}$ is the concentration of the i-th species, $n_{i}=$ number of Bub1 units bound to i-th species,

$732[\text { BubR1 }]_{\mathrm{T}}=100 \mathrm{nM}$. The equilibrium concentration of each state was obtained by numerically 733 solving $\dot{\mathbf{X}}=\dot{y}_{i}=0, i=1,2 \ldots, 5$.

734 In experiments, the concentration of eSAC activator complex is measured in arbitrary units of 735 mCherry fluorescence (a. u.), whereas in our model the unit of concentration is nano moles (nM). 736 In our simulations, we chose the maximum value of concentration of eSAC activator complex (the 737 value corresponding to $20 \mathrm{a}$. $\mathrm{u}$. in experiments) to be $200 \mathrm{nM}$. For easier comparison to 738 experimental figures, in our simulation results the eSAC activator complex concentration is expressed in arbitrary units, with $1 \mathrm{a}$. $\mathrm{u}$. of fluorescence corresponding to $10 \mathrm{nM}$.

741 Figure S3A shows the abundance of different Bub1 and BubR1-bound states as functions of the 742 total concentration of eSAC activator complex for $\mathrm{KI} 1^{*}-\mathrm{KI} 2^{*}$. At low eSAC concentrations, the 743 eSAC tends to be highly loaded, with Bub1 and BubR1 on every MELT motif. However, for cells 
744

745

746

747

748

749

750

751

752

753

754

755

756

757

758

759

760

761

762

763

764

765

766

767

768

769

770

771

with a high eSAC concentration, [eSAC] $>>[B u b 1]_{T}$, the most abundant eSAC species is one that does not bind any Bub1 at all (not shown), followed by species that bind either Bub1 or Bub1BubR1 at only one of the MELT motifs. We define the sum of concentration of MELPT-Bub1 and MELpT-Bub1-BubR1 as [eSAC] $\mathrm{T}$ :

$$
\begin{gathered}
{[\mathrm{eSAC}]_{\mathrm{T}}=[\text { MELpT-Bub1 }]+[\text { MELpT-Bub1-BubR1 }]} \\
{[\text { MELpT-Bub1 }]=\sum_{i=1}^{N}\left(n_{i}-m_{i}\right) \cdot x_{i}} \\
{[\text { MELpT-Bub1-BubR1 }]=\sum_{i=1}^{N} m_{i} \cdot x_{i}}
\end{gathered}
$$

\section{Formation of MCC by the eSAC signaling complexes}

We assume that the recruitment of SAC proteins (Bub1 and BubR1) to MELT motifs of the eSAC activator complexes enables the incorporation of Cd20 into MCC. Since different species catalyze this reaction at different rates, we define the effective rate of conversion, $k_{\text {asmcc }}$, as the concentration-weighted sum of the conversion rates of each eSAC complex:

$$
k_{\mathrm{asmcc}}=\frac{1}{[\mathrm{eSAC}]_{\mathrm{T}}} \cdot\left[k_{\mathrm{MCC}} \cdot \sum_{i=1}^{N}\left(n_{i}-m_{i}\right) \cdot x_{i}+k_{\mathrm{MCC}}^{\prime} \cdot \sum_{i=1}^{N} m_{i} \cdot x_{i}\right] \text {, }
$$

where $k_{\mathrm{MCC}}$ and $k_{\mathrm{MCC}}^{\prime}$ are the MCC formation rates due to MELpT-Bub1 and MELpT-Bub1BubR1, respectively. Note, for most species, the rate of MCC generation is additive. For example, $k_{0012}=k_{\mathrm{MCC}}+k_{\mathrm{MCC}}^{\prime}$ for a phosphodomain that binds only Bub1 at the $13^{\text {th }}$ MELT motif and Bub1-BubR1 at $14^{\text {th }}$ MELT motif (Table S3). Using $[\mathrm{eSAC}]_{\mathrm{T}}$ and $k_{\text {asmcc }}$ as inputs for the He model (discussed below), we calculated the time evolution of Cyclin B concentration and from it the time in mitosis.

\section{Stage 2: The effect of MCC produced on exit from mitosis}

To calculate the effect of MCC generated by the eSAC on mitotic exit, we used a simplified version of the model of the mitotic checkpoint proposed by the He model (He et al., 2011). Active eSAC signaling complexes $\left(\mathrm{eSAC}_{\mathrm{A}}\right)$ generate $\mathrm{MCC}$, as described in the previous section. The temporal dynamics of our mitotic checkpoint model are determined by the ordinary differential equations:

$$
\frac{\mathrm{d}[\mathrm{CycB}]}{\mathrm{d} t}=k_{\mathrm{scyc}}-\left(k_{\mathrm{dcyc}}+k_{\mathrm{dcyc}, \mathrm{c} 20} \cdot[\mathrm{Cdc} 20]\right) \cdot[\mathrm{CycB}]
$$




$$
\begin{array}{r}
\frac{\mathrm{d}\left[\mathrm{eSAC}_{\mathrm{A}}\right]}{\mathrm{d} t}=k_{\mathrm{ancyc}} \cdot[\mathrm{CycB}] \cdot\left([\mathrm{eSAC}]_{\mathrm{T}}-\left[\mathrm{eSAC}_{\mathrm{A}}\right]\right)-k_{\mathrm{in}, \mathrm{capp}} \cdot[\mathrm{CAPP}] \cdot\left[\mathrm{eSAC}_{\mathrm{A}}\right] \\
\frac{\mathrm{d}[\mathrm{MCC}]}{\mathrm{d} t}=k_{\mathrm{asmcc}} \cdot[\mathrm{Cdc} 20] \cdot[\mathrm{eSAC}]_{\mathrm{A}}-\left(k_{\mathrm{dimcc}}+k_{\mathrm{imad}}+k_{\mathrm{imad}, \mathrm{c} 20} \cdot[\mathrm{Cdc} 20]\right) \cdot[\mathrm{MCC}] \\
\frac{\mathrm{d}[\mathrm{CAPP}]}{\mathrm{d} t}=k_{\mathrm{acapp}} \cdot\left([\mathrm{CAPP}]_{\mathrm{T}}-[\mathrm{CAPP}]\right)-k_{\text {icapp,cyc }} \cdot[\mathrm{CycB}] \cdot[\mathrm{CAPP}]
\end{array}
$$

775

In these equations, $[\mathrm{CycB}]=[\mathrm{CDK}-\mathrm{CycB}],[\mathrm{Cdc} 20]=[\mathrm{APC}-\mathrm{Cdc} 20],[\mathrm{eSAC}]_{\mathrm{T}}$ is the total concentration of Bub1 and BubR1-bound eSAC signaling complexes (Eq. 10), which is either in the active, signaling-competent state $\mathrm{eSAC}_{\mathrm{A}}$, or the inactive state, $\left[\mathrm{eSAC}_{\mathrm{I}}\right]=[\mathrm{eSAC}]_{\mathrm{T}}-\left[\mathrm{eSAC}_{\mathrm{A}}\right]$. [MCC] and [CAPP] refer to the concentrations of the mitotic checkpoint complex and the CDKcounteracting protein phosphatase, respectively. In addition, the total concentration of Cdc20 is:

$$
[\mathrm{Cdc} 20]_{\mathrm{T}}=[\mathrm{Cdc} 20]+[\mathrm{MCC}]
$$

Here, the subscript $T$ indicates total concentration. The values of the parameters in the model and of the fixed concentrations of some components, as listed in Table S2, are taken from (He et al., 2011).

Simulation of time in mitosis

To simulate 'time in mitosis', we assume that a cell exits mitosis when [CycB] drops below $1 \mathrm{nM}$. We numerically integrated Ordinary Differential Equations (ODEs) to calculate the time evolution of $[\mathrm{CycB}]$ and the other components of the mitotic checkpoint. As before, the initial conditions for the simulation are chosen to be $[\mathrm{CycB}]=45 \mathrm{nM},\left[\mathrm{eSAC}_{\mathrm{A}}\right]=0,[\mathrm{MCC}]=25 \mathrm{nM}$, and $[\mathrm{CAPP}]=5$ $\mathrm{nM}$. The qualitative aspects of our results do not change if the initial conditions are changed a

793 little bit. Figure S3C displays typical time courses for [CycB], for different eSACs, for [eSAC 794 activator complex] = 10 a. u. The system always comes out of mitosis (as seen by the drop in $795[\mathrm{CycB}])$, albeit after different time delays.

\section{Statistical analysis}

798 To determine the overall trend in the dose-response data, the data were first binned (in MATLAB) 799 and then the mean values of each bin were overlayed on the data. The number of observations, 800 technical replicates are noted in the figure legends. These mean values were fit with a fourparameter sigmoidal curve using the GraphPad Prism 9 software. Statistical significance of the difference between the mean values in Figure 5B was assessed using the unpaired t-test with 
Figure 1

A

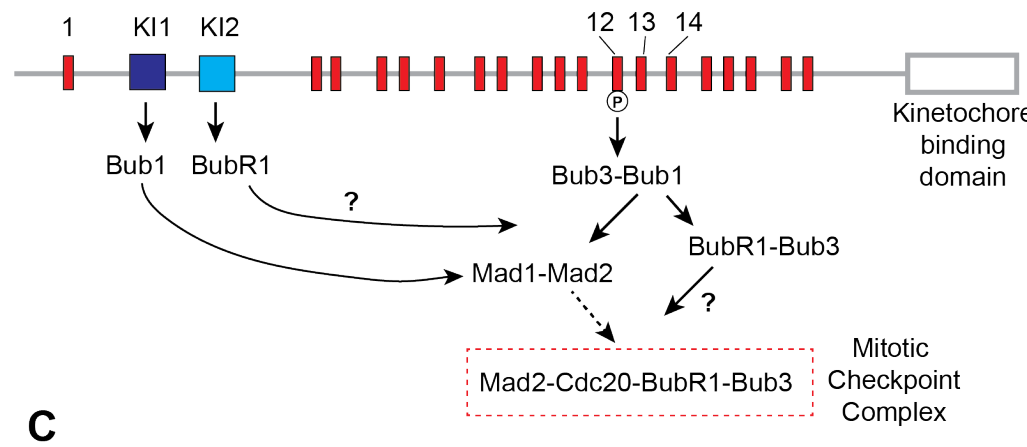

B
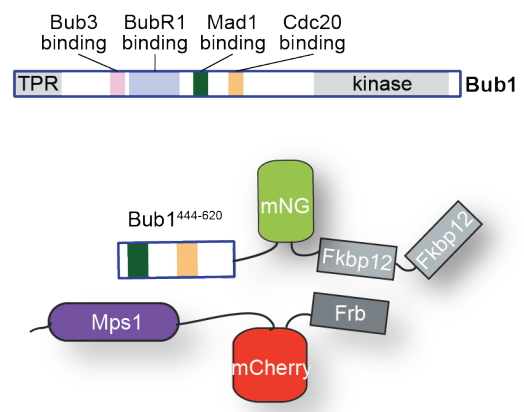

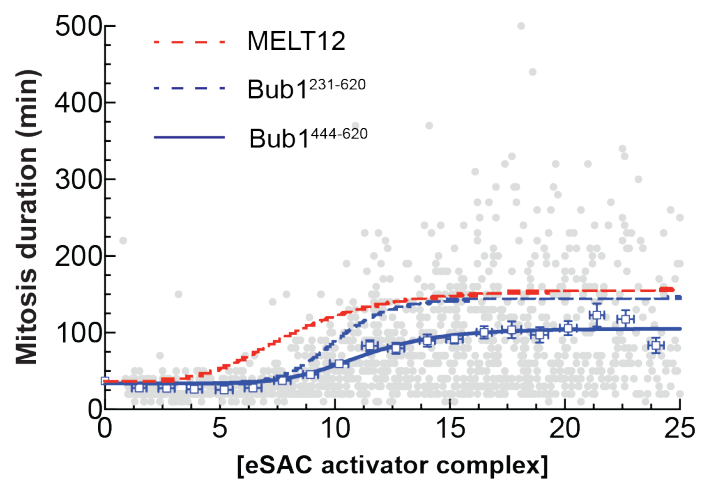

D

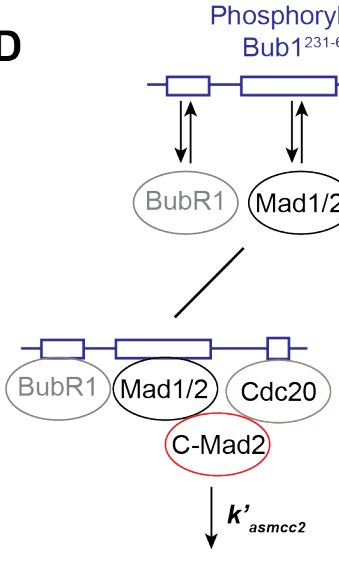

Mitotic Checkpoint
complex

Figure 1 The contribution of Bub1-BubR1 heterodimerization to MCC assembly during eSAC signaling. A Schematic at the top displays the domain organization of human Knl1. Below is a simplified schematic displaying the pathways that recruit SAC signaling proteins to unattached kinetochores. B Schematic at the top displays the domain organization of human Bub1. The 
810 cartoon represents the ectopic SAC activation or 'eSAC' system. C Dose-response data for an

811 eSAC system that uses Bub1 $1^{444-620}$ as the phosphodomains. Each gray circle displays the average

812 cellular mCherry fluorescence ( $X$ axis) and the time in mitosis ( $Y$ axis) for a single cell $(n=1920$

813 from 2 experiments; 139 and 155 are the lower and upper 95\% confidence intervals). Blue

814 squares represent mean values of the dose-response data binned according to mCherry

815 fluorescence. Vertical and horizontal lines represent S.E.M. The solid blue curve displays the

816 least squares 4-parameter sigmoidal fit to the binned mean values. Sigmoidal fits for dose-

817 response data for one MELT motif (MELT12, dashed red curve) and an extended Bub1 ${ }^{231-620}$

818 phosphodomain containing the Bub3- and BubR1-binding sites (dashed blue curve) from our

819 previous study have been super-imposed for comparison (Chen et al., 2019). Bar graph on the

820 right displays the maximal mitotic duration estimated by the sigmoidal fit. Vertical lines represent

$82195 \%$ confidence interval on the fit parameter. D Cartoon displays the simplified scheme used to

822 simulate the generation of MCC by the eSAC system. E Numerical simulation of the dose-

823 response curves by assuming the assembly of signaling complexes and ultimately the MCC as

824 show in $\mathbf{D}$ followed by a bi-stable switch based on negative feedback described by $\mathrm{He}$ et al ( $\mathrm{He}$

825 et al., 2011).

826

827

828 
A
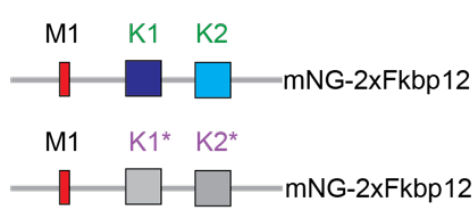
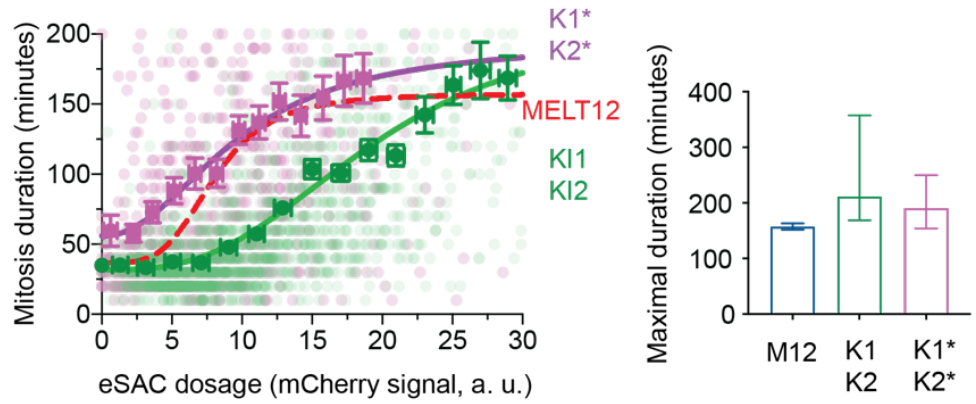

B
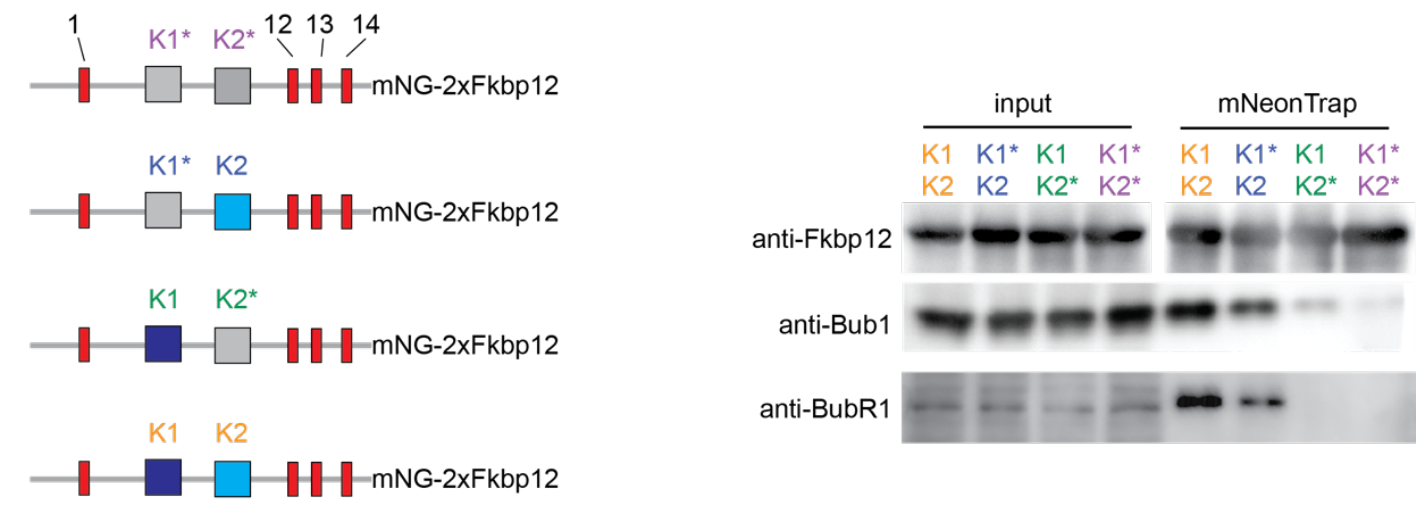

C
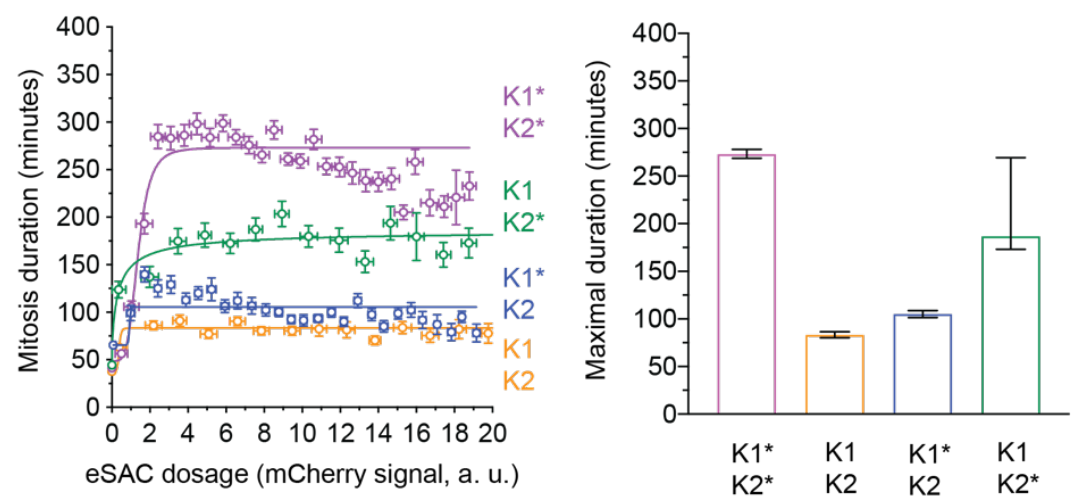

Figure 2 Recruitment of Bub1 and BubR1 by the KI motifs in KNL1 and their contribution to eSAC signaling. A Left: Schematic of the two phosphodomains used to test whether the $\mathrm{KI}$ motifs contribute to MCC assembly mediated by the MELT motif in the eSAC phosphodomain. $\mathrm{KI} 1-\mathrm{KI} 2$ indicates phosphodomain with intact $\mathrm{KI}$ motifs, $\mathrm{KI} 1^{*}-\mathrm{KI}{ }^{*}$ indicates phosphodomains wherein the $\mathrm{KI}$ motifs are inactivated using suitable point mutations (see Methods for details). Scatter plot in the middle displays the dose-response data for the two phosphodomains $(\mathrm{n}=1888$ in green and $\mathrm{n}=836$ in magenta from $\geq 2$ experiments respectively for $\mathrm{KI} 1-\mathrm{KI} 2$ and $\mathrm{KI} 1^{*}-\mathrm{KI} 2^{*}$; 
839 maximal mitotic duration predicted by 4-parameter sigmoidal fits to the binned mean values as in

840 Figure 1C. Vertical lines display 95\% confidence intervals on the fit parameter. B Left: Schematic 841 of the phosphodomains consisting of 4 MELT motifs and either active or inactive (indicated by *)

842 KI motifs. Right: Immunoprecipitation of the eSAC phosphodomains using mNeonGreenTrap 843 beads followed by Western blot analysis to probe for the co-IP of the indicated proteins. C Dose844 response data for the indicated phosphodomains. Only the binned mean values are shown for 845 clarity. Statistical analysis performed as in 1D ( $\mathrm{n}=1019,1024,666$, and 3219 for KI1-KI2, KI1$846 \mathrm{KI} 2^{*}, \mathrm{KI} 1^{*}-\mathrm{K} 2$, and $\mathrm{KI} 1^{*}-\mathrm{KI} 2^{*}$ respectively from $\geq 2$ experiments). Bar graph on the right displays 847 the maximal time in mitosis predicted by the sigmoidal fits. 
A Stage 1 - Calculate equilibrium concentrations for signaling complexes

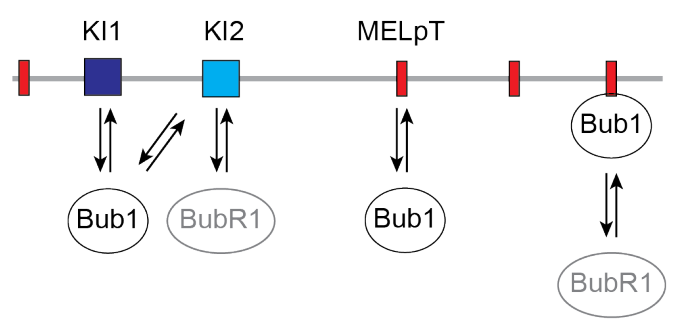

B
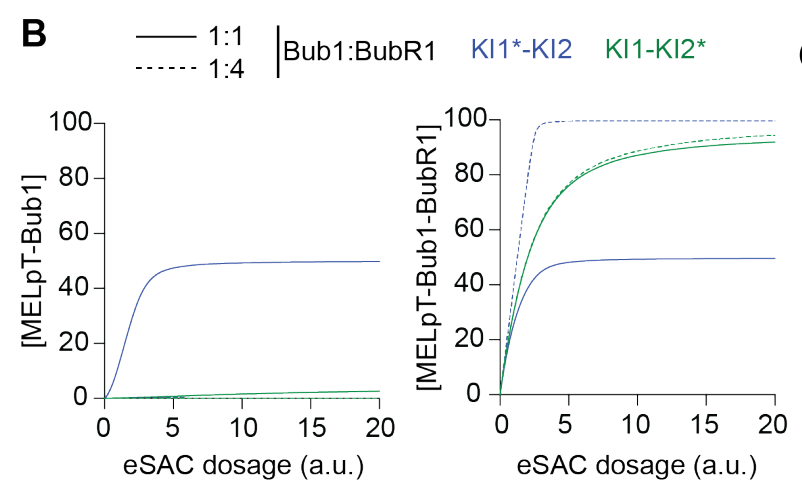

D

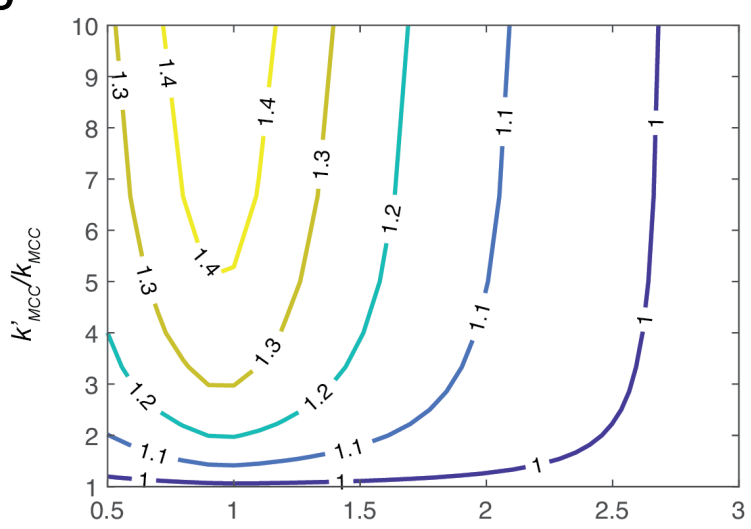

850

851

852

853

854

855

856

857

858
Stage 2 - Calculate steady-state MCC concentration and its effect on cyclin B degradation and anaphase onset

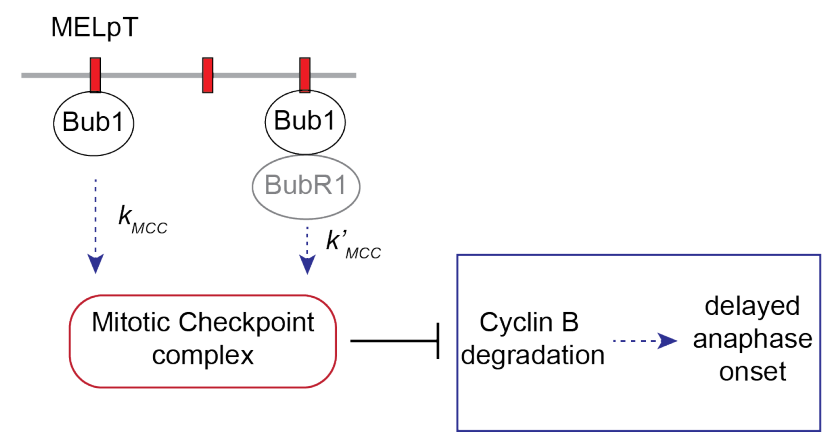

C

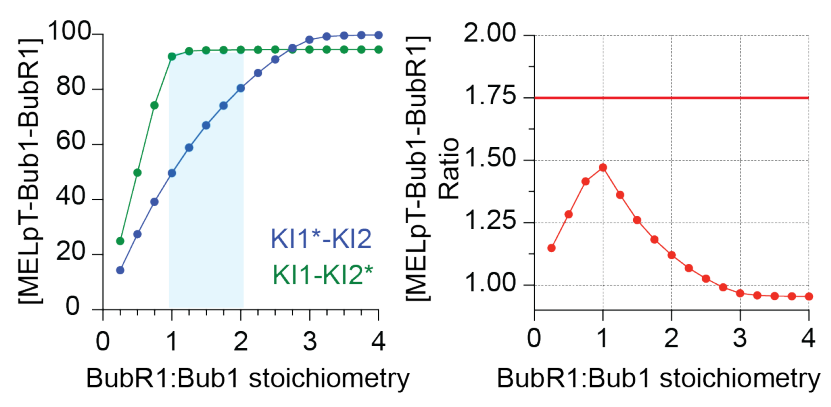

E

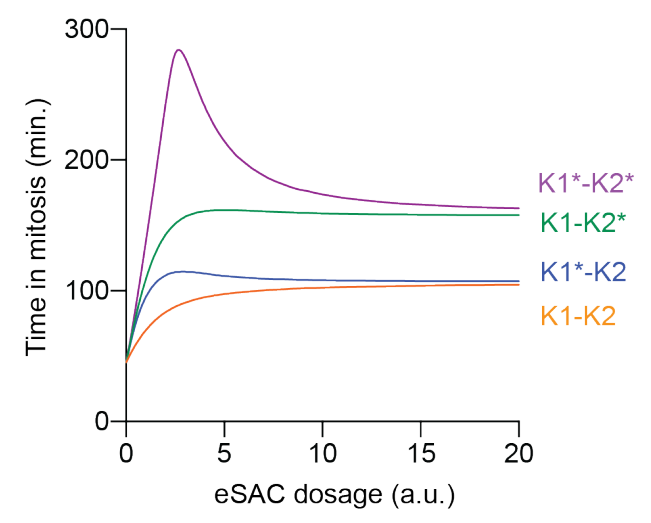

Figure 3 Numerical simulation of the dose-response data for the eSAC phosphodomains containing KI motifs. A Schematic of the two-stage model used to simulate the dose-response curves. B The influence of Bub1-BubR1 stoichiometry and the differential depletion of Bub1 and BubR1 by the $\mathrm{KI}$ motifs in the simulations on the equilibrium concentrations of the two signaling complexes formed on phosphorylated MELT motifs. C Comparison of the equilibrium concentration of the MELpT-Bub1-BubR1 complex assembled by $\mathrm{KI} 1^{*}-\mathrm{KI} 2$ and $\mathrm{KI} 1-\mathrm{KI}{ }^{*}$ (left) and their ratio (right) as a function of the Bub1-BubR1 stoichiometry. For the two phosphodomains to generate different responses, the Bub1-BubR1 stoichiometry must be around 1:1 (indicated by 
859 the blue shaded area). D Contour plot shows the ratio of the maximal responses produced by $860 \mathrm{KI} 1^{*}-\mathrm{KI} 2$ and $\mathrm{KI} 1-\mathrm{KI} 2^{*}$ as a function of the Bub1-BubR1 stoichiometry and the ratio of the rates at 861 which the MELpT-Bub1-BubR1 and the MELpT-Bub1 signaling complexes produce MCC (i.e., $\left.862 k_{M C}^{\prime} / k_{M C C}\right)$. E Simulation of the dose-response curves for the four phosphodomains using the 863 same set of parameter values. 
Figure 4
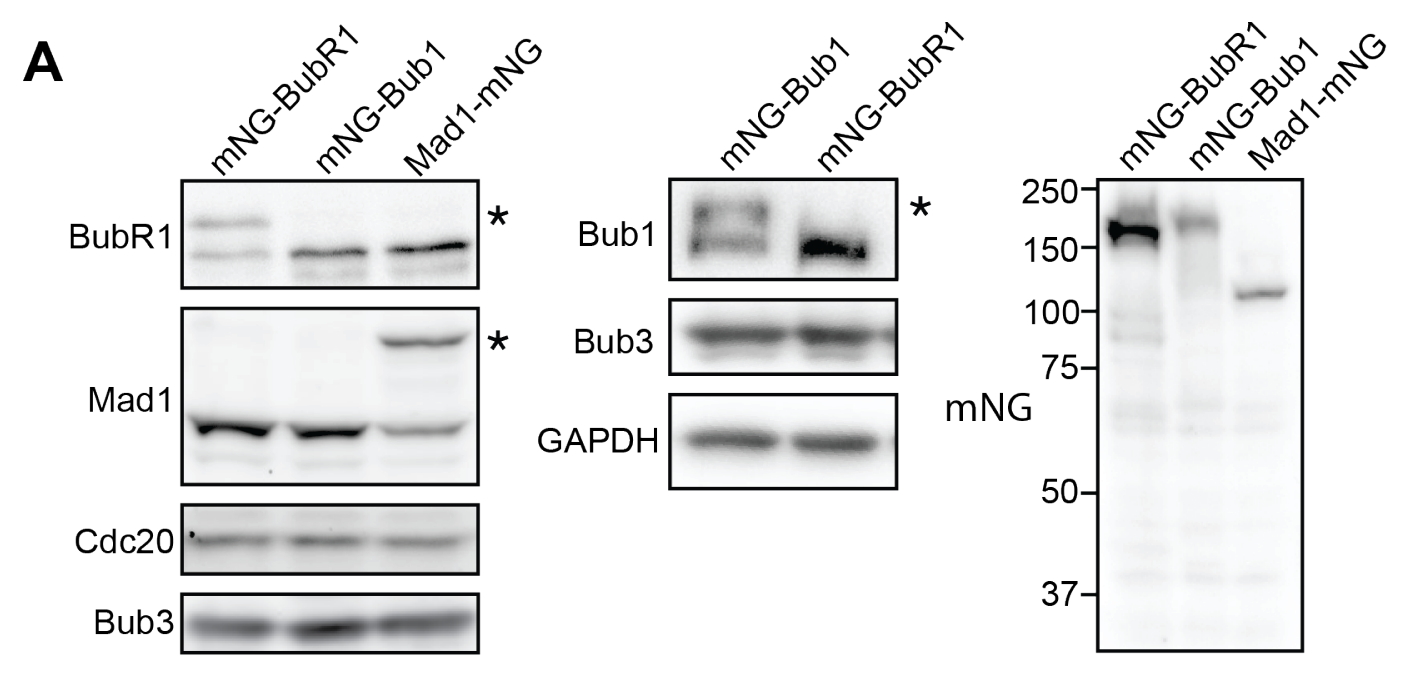

B
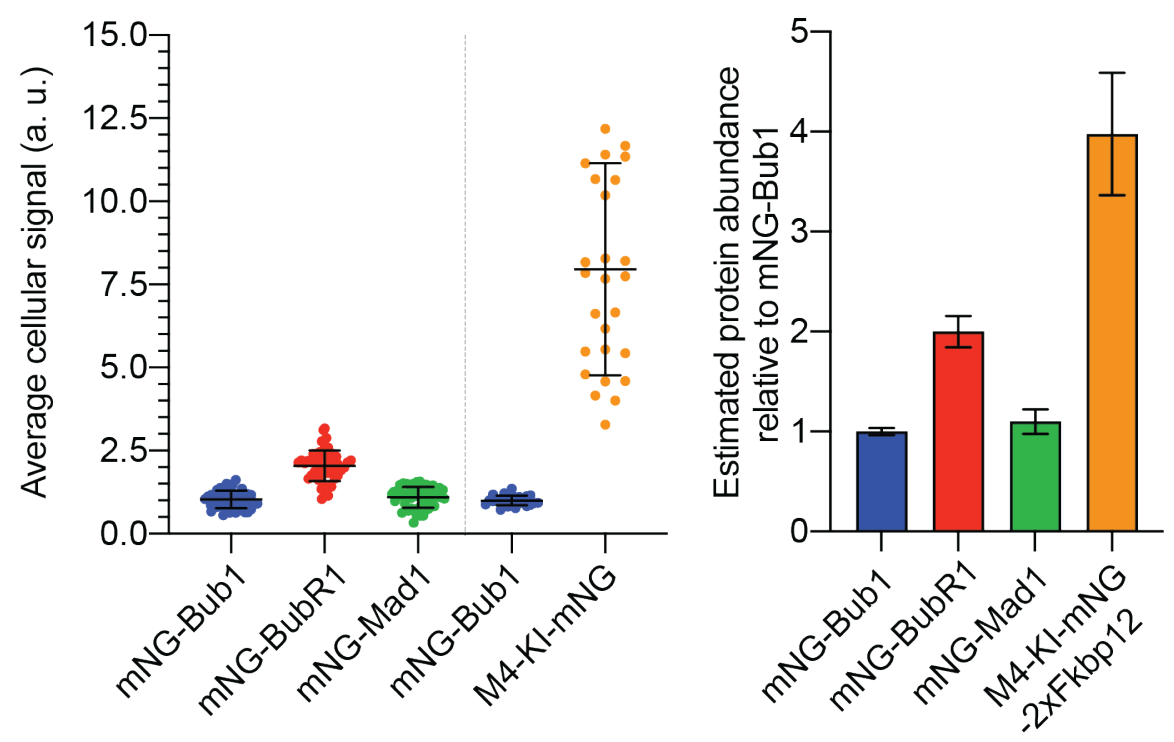

Figure 4 Generation of CRISPR/Cas9 genome-edited HeLa cell lines expressing mNeonGreen-Bub1, mNeonGreen-BubR1, and Mad1-mNeonGreen. A Western blots show that showing that roughly half the BubR1 and Mad1 (left) and Bub1 (middle) is tagged with $\mathrm{mNeonGreen} \mathrm{in} \mathrm{the} \mathrm{three} \mathrm{partially} \mathrm{genome-edited} \mathrm{cell} \mathrm{lines} \mathrm{(asterisks} \mathrm{on} \mathrm{the} \mathrm{right} \mathrm{of} \mathrm{each}$

871 displayed blot mark the mNG fusion protein). Western blot on the right shows whole cell extracts

872 of the three cell lines probed with anti-mNeonGreen antibodies. B Average mNG-Bub1, mNG-

873 BubR1, Mad1-mNG, and M4-KI1-KI2-mNG-2xFkbp12 signals from mitotic HeLa cells (left) and 874 estimation of the relative protein abundance, assuming that the total protein is twice as high as 875 the abundance of the $\mathrm{mNG}$-labeled species (mean \pm std. dev.). 
A
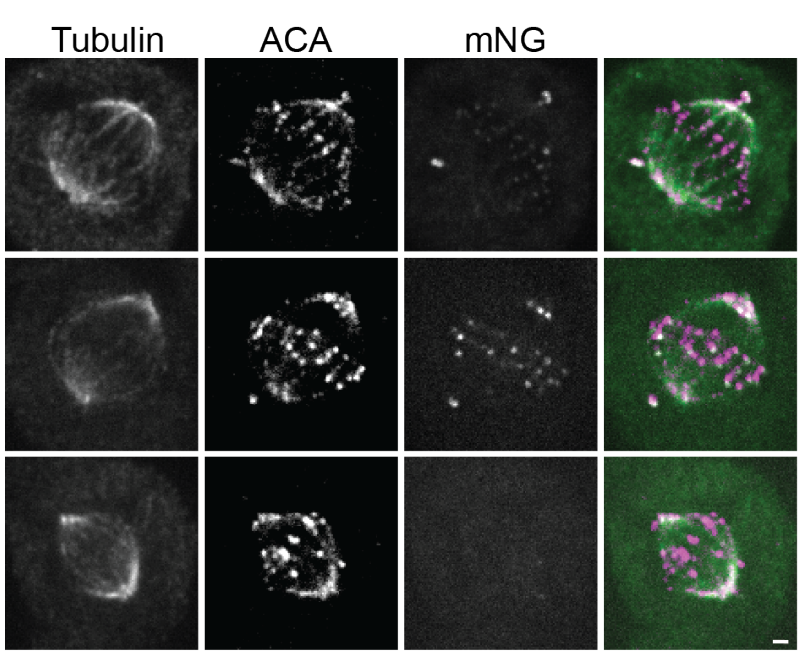

B
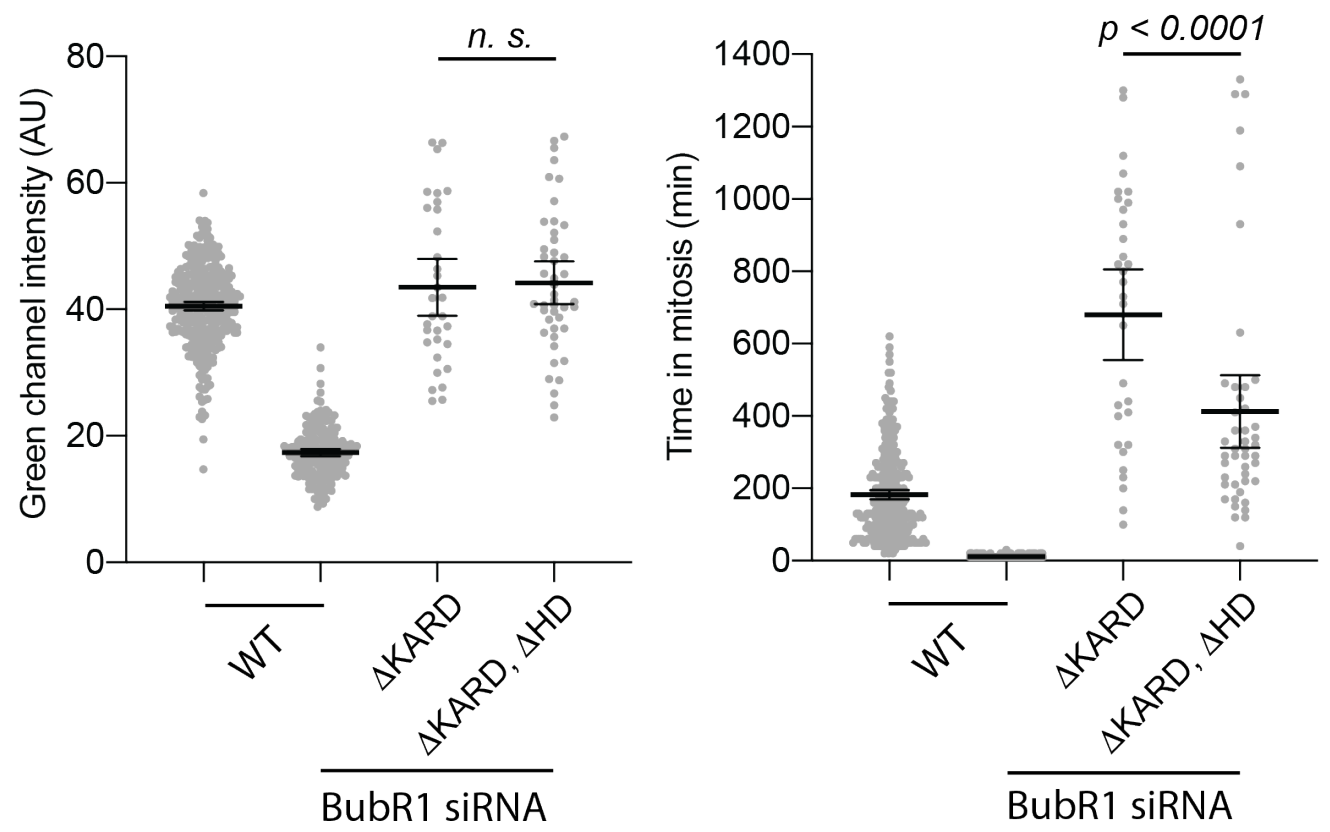

Figure 5 Recruitment of BubR1 by BuB1 per se contributes to the signaling strength of the

SAC. A Micrographs of representative cells displaying the indicated antigens at the top. The top row displays micrographs of the partially genome-edited cell line expressing mNG-BubR1. The bottom two rows display representative cells after endogenous BubR1 was knocked down and the indicated mutant, mNG-tagged version was ectopically expressed (scale bar $\sim 1.22$ microns).

B Scatterplot on the left displays the duration of mitosis of either genome-edited cells expressing

WT BubR1 or cells that ectopically expressed the indicated mutant after BubR1 RNAi in media containing $50 \mathrm{nM}$ nocodazole. Scatterplot on the right displays the average intracellular $\mathrm{mNeonGreen} \mathrm{signal} \mathrm{from} \mathrm{the} \mathrm{same} \mathrm{cells} \mathrm{(mean} \pm 95 \%$ confidence intervals). 


\section{Supplementary Figure 1}

A

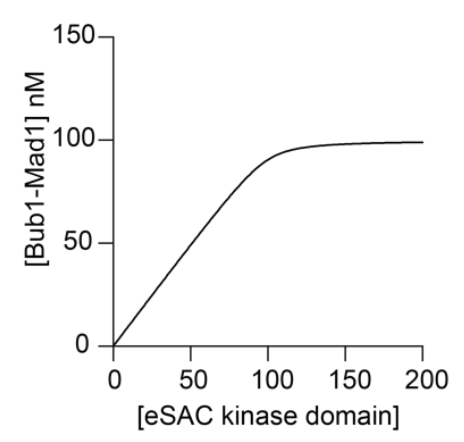

C

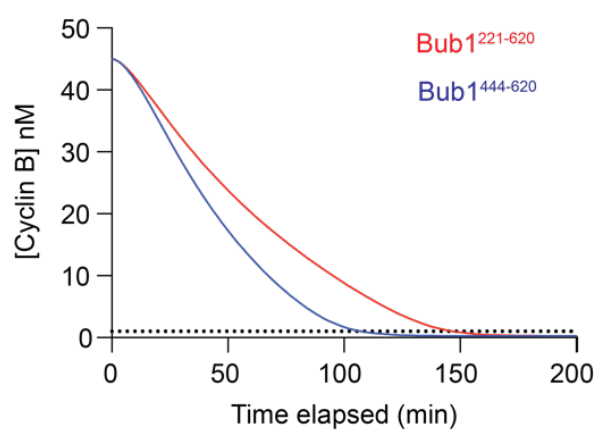

E

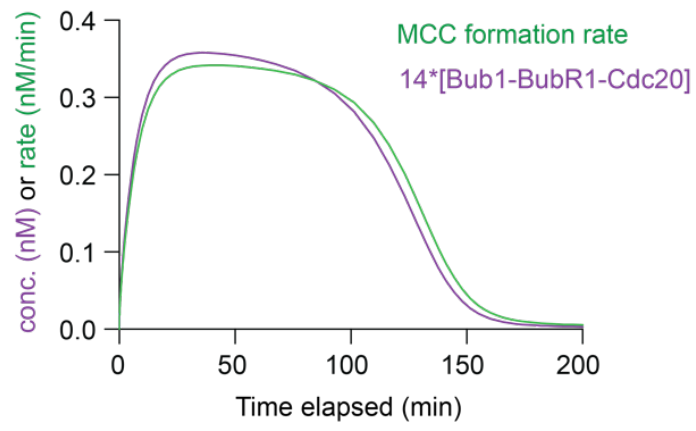

B

D
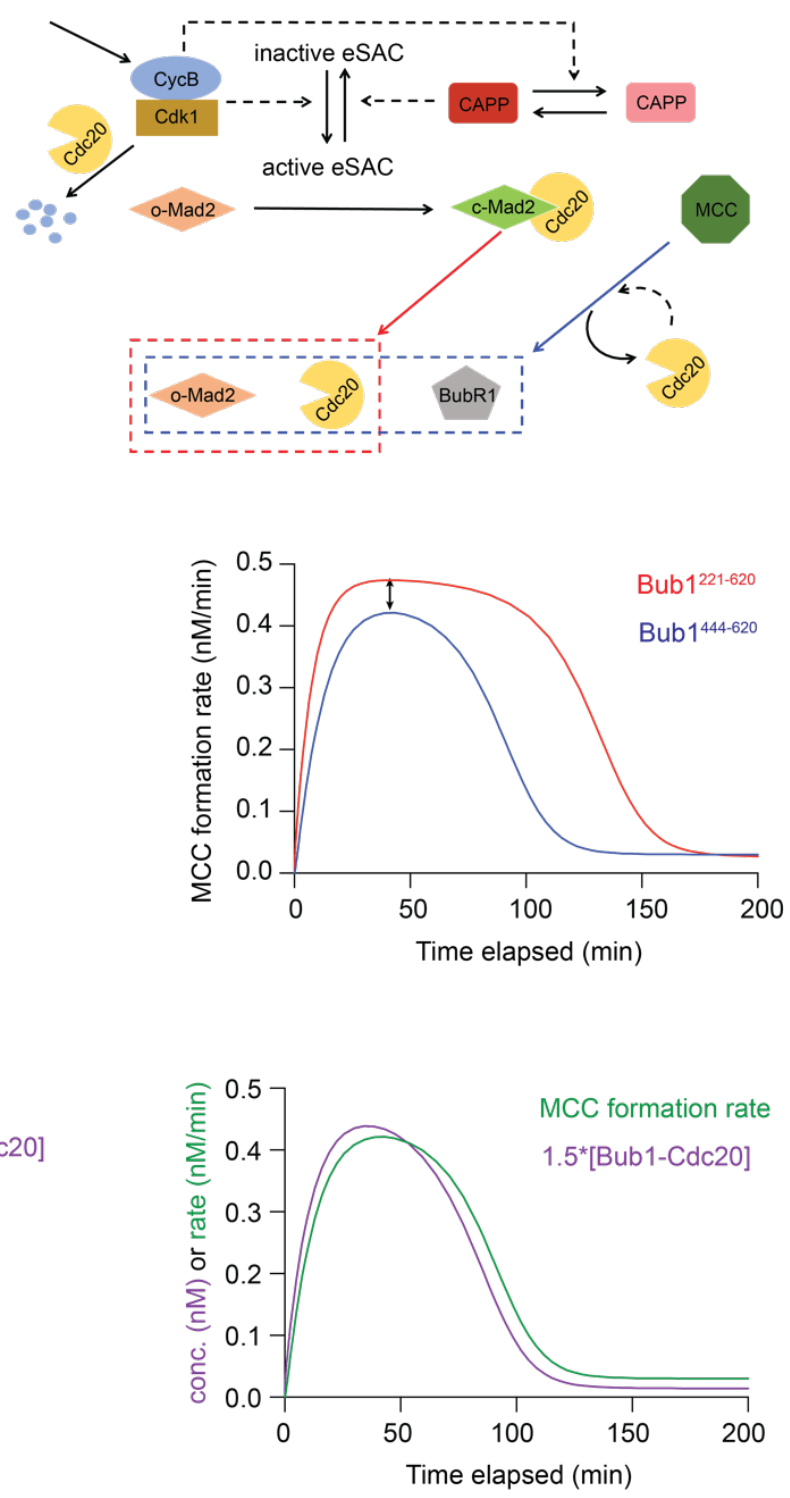

Figure S1 Simulation of the dose-response curves for the Bub1 eSAC system. A The amount of the eSAC signaling complex (Bub1-Mad1) saturates due to the limited availability of Mad1 (100 nM). B Schematic of the model used to simulate the dose-response data for the "full" and "truncated" Bub1 phosphodomain used in the eSAC system. C Temporal evolution of Cyclin B concentration for the two eSAC systems. D Assessment of MCC formation rates for the full and truncated phosphodomain. E Superimposition of the dynamics of the MCC formation rate and the concentration of signaling complexes that produce MCC. 898 


\section{Supplementary Figure 2}

A

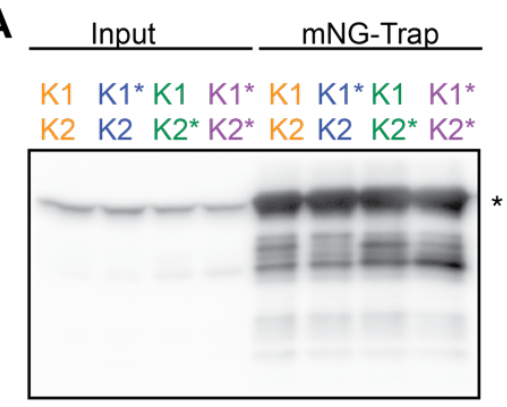

Anti-FKBP12

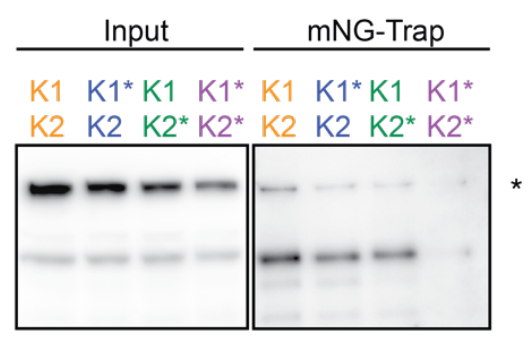

Anti-Bub1

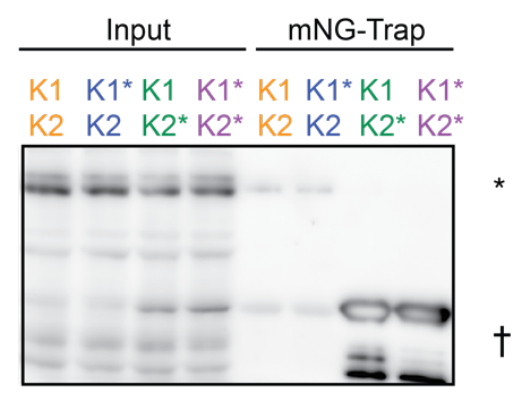

Anti-BubR1

B
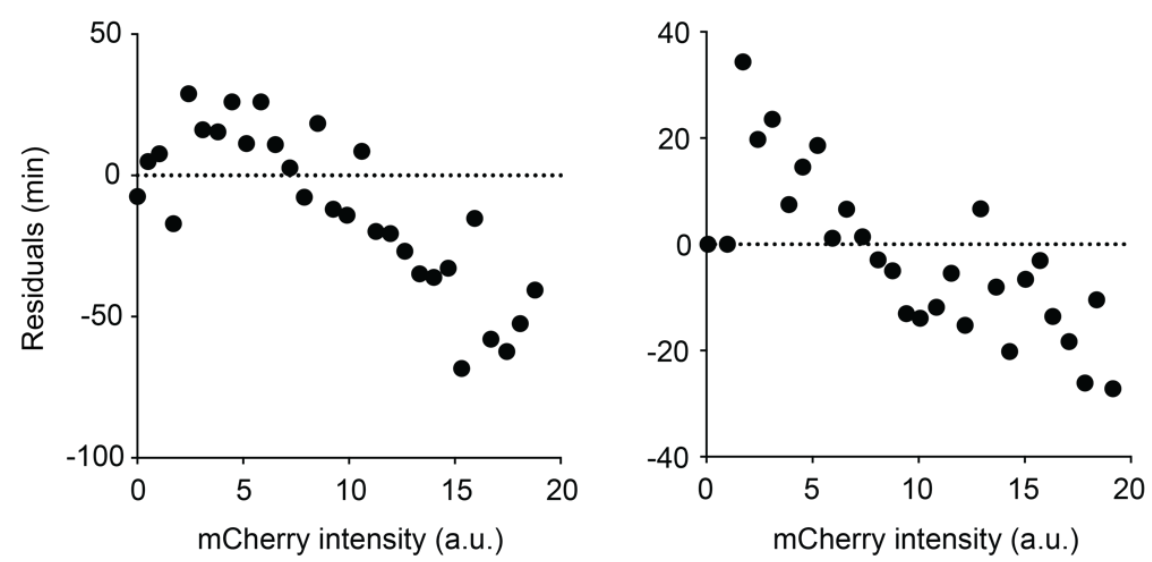

C

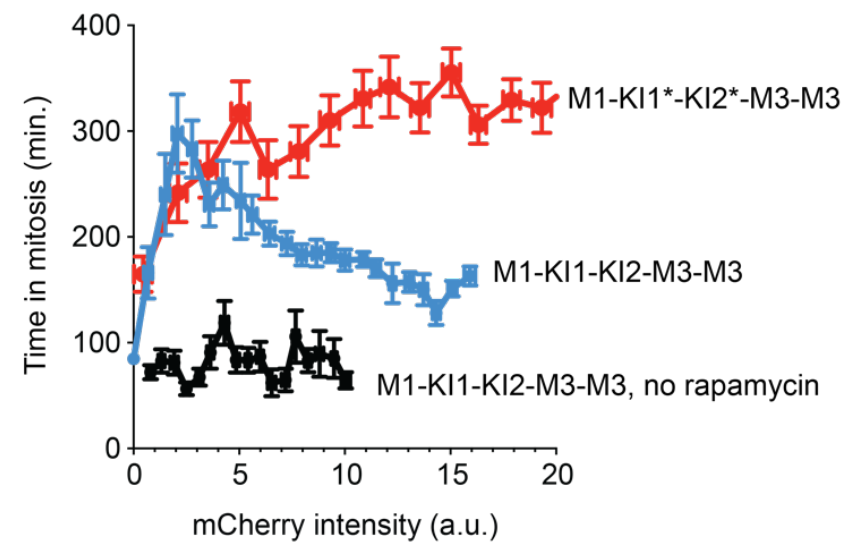

Supplementary Figure 2 A Western blot probing for the indicated protein for assessing the coimmunoprecipitation of Bub1 and BubR1 with the indicated eSAC phosphodomain (at the top). The asterisk marks the position of the band corresponding to the full-length protein. The cross marks bands that likely degradation products of the full-length protein. B Residual plots from a 4- 
908 parameter sigmoidal fit to the dose-response data for the $\mathrm{KI}{ }^{*}-\mathrm{KI}{ }^{*}$ (left) and $\mathrm{KI} 1-\mathrm{KI}{ }^{*}$ (right) 909 phosphodomains. C Dose-response data for the indicated phosphodomains. Circles represent 910 average values of binned data (vertical and horizontal lines display standard error on each mean 911 value. $\mathrm{n}=498,1313$, and 394 respectively for M1-KI1-KI2-M3-M3, M1-KI1*-KI2*-M3-M3, and M1$912 \mathrm{KI} 1-\mathrm{KI} 2-\mathrm{M} 3-\mathrm{M} 3$ without rapamycin respectively pooled from two or more experiments). 
A

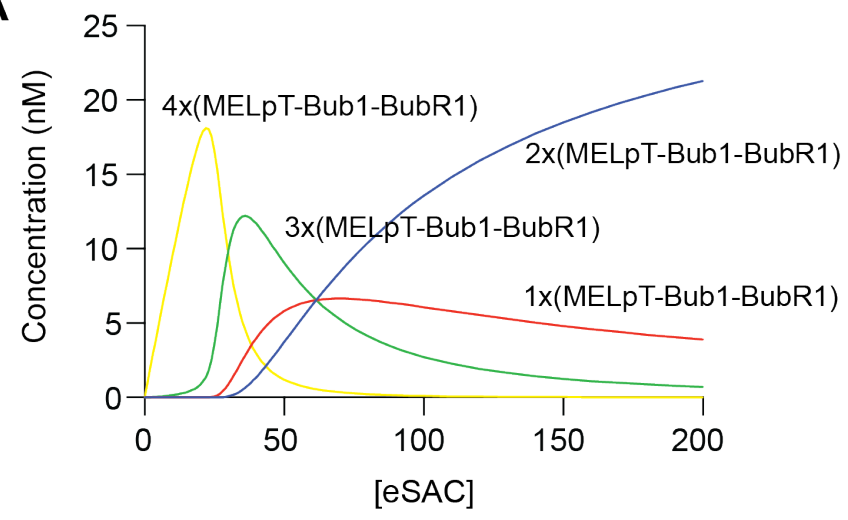

C

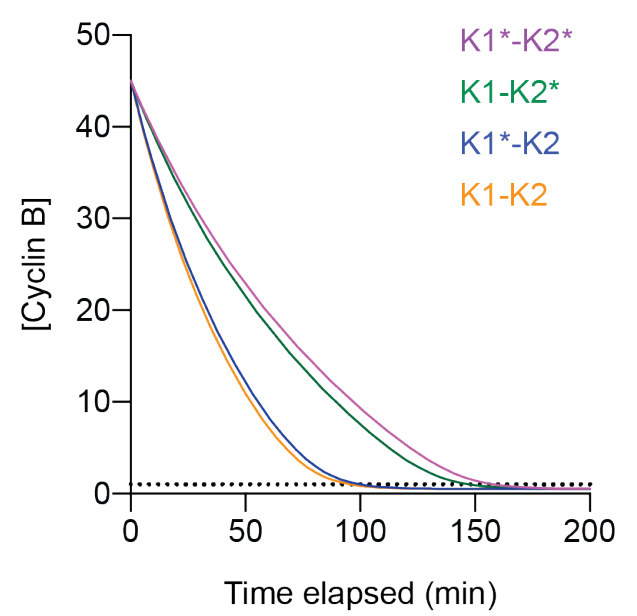

B

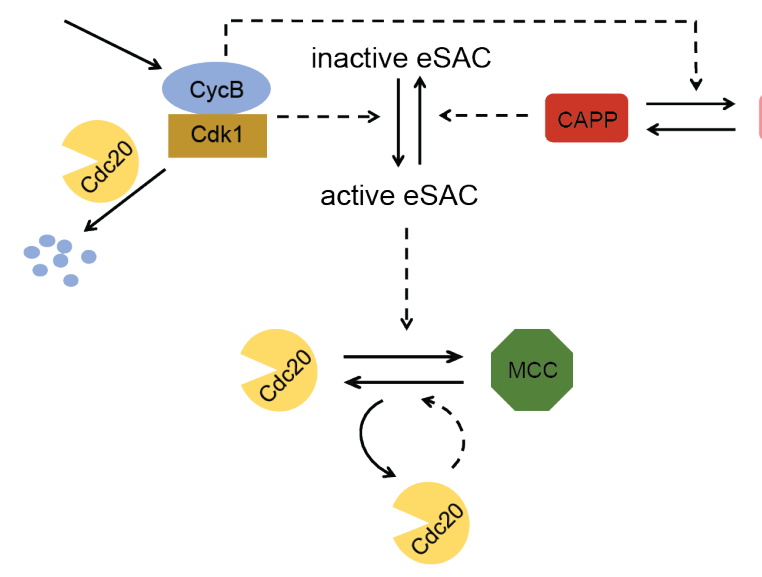

D

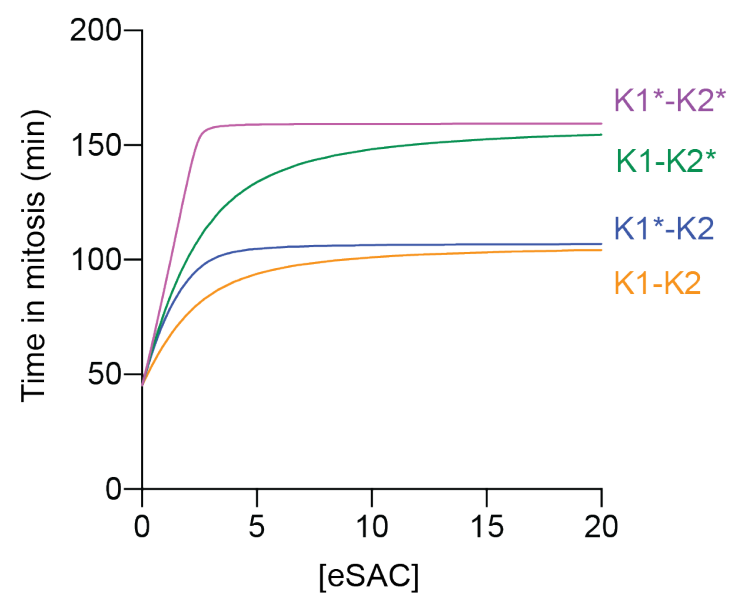
and MELT motifs. A Abundance of the indicated eSAC signaling complex (of the form $\mathrm{n}^{*}$ (MELpTBub1-BubR1)) as a function of the total concentration of the eSAC activator complex. The number

$921 \mathrm{n}$ indicates the number of MELT motifs bound to Bub1-BubR1. Also see Table S3. B. Schematic

922 of the model used to simulate the dose-response data for ESAC with MELT and KI motifs. C

923 Temporal evolution of Cyclin B concentration for the MELT and KI motif eSAC system. D

924 Simulation of dose-response curves for the four phosphodomains without synergistic interactions 925 between the MELT motifs. 


\section{References}

928 Bolanos-Garcia, V.M., Lischetti, T., Matak-Vinkovic, D., Cota, E., Simpson, P.J., Chirgadze, D.Y., 929 Spring, D.R., Robinson, C.V., Nilsson, J., and Blundell, T.L. (2011a). Structure of a Blinkin-BUBR1 930 complex reveals an interaction crucial for kinetochore-mitotic checkpoint regulation via an 931 unanticipated binding Site. Structure 19, 1691-1700.

932 Bolanos-Garcia, Victor M., Lischetti, T., Matak-Vinković, D., Cota, E., Simpson, Pete J., Chirgadze, 933 Dimitri Y., Spring, David R., Robinson, Carol V., Nilsson, J., and Blundell, Tom L. (2011b). Structure 934 of a Blinkin-BUBR1 Complex Reveals an Interaction Crucial for Kinetochore-Mitotic Checkpoint Regulation via an Unanticipated Binding Site. Structure 19, 1691-1700. Bouchoux, C., and UhImann, F. (2011). A quantitative model for ordered Cdk substrate dephosphorylation during mitotic exit. Cell 147, 803-814. Chen, C., Whitney, I.P., Banerjee, A., Sacristan, C., Sekhri, P., Kern, D.M., Fontan, A., Kops, G., Tyson, J.J., Cheeseman, I.M., et al. (2019). Ectopic Activation of the Spindle Assembly Checkpoint Signaling Cascade Reveals Its Biochemical Design. Curr Biol 29, 104-119 e110.

941 Collin, P., Nashchekina, O., Walker, R., and Pines, J. (2013). The spindle assembly checkpoint works like a rheostat rather than a toggle switch. Nat Cell Biol 15, 1378-1385.

944 Dick, A.E., and Gerlich, D.W. (2013). Kinetic framework of spindle assembly checkpoint signalling. Nat Cell Biol 15, 1370-1377. Musacchio, A. (2017). Basis of catalytic assembly of the mitotic checkpoint complex. Nature. Foley, E.A., Maldonado, M., and Kapoor, T.M. (2011). Formation of stable attachments between kinetochores and microtubules depends on the B56-PP2A phosphatase. Nat Cell Biol 13, 12651271. He, E., Kapuy, O., Oliveira, R.A., Uhlmann, F., Tyson, J.J., and Novák, B. (2011). System-level feedbacks make the anaphase switch irreversible. Proceedings of the National Academy of Sciences. Ji, Z., Gao, H., Jia, L., Li, B., and Yu, H. (2017). A sequential multi-target Mps1 phosphorylation cascade promotes spindle checkpoint signaling. Elife 6 .

Jia, L., Li, B., and Yu, H. (2016). The Bub1-Plk1 kinase complex promotes spindle checkpoint signalling through Cdc20 phosphorylation. Nat Commun 7, 10818.

Khandelia, P., Yap, K., and Makeyev, E.V. (2011). Streamlined platform for short hairpin RNA interference and transgenesis in cultured mammalian cells. Proc Natl Acad Sci USA 108, 1279912804. Kiyomitsu, T., Murakami, H., and Yanagida, M. (2011). Protein interaction domain mapping of human kinetochore protein Blinkin reveals a consensus motif for binding of spindle assembly checkpoint proteins Bub1 and BubR1. Mol Cell Biol 31, 998-1011. $\mathrm{Knl} 1$ enhance assembly of comprehensive spindle checkpoint complexes around MELT repeats. Curr Biol 24, 29-39.

967 Krenn, V., Wehenkel, A., Li, X., Santaguida, S., and Musacchio, A. (2012). Structural analysis reveals features of the spindle checkpoint kinase Bub1-kinetochore subunit Knl1 interaction. J Cell Biol 196, 451-467. 
Lara-Gonzalez, P., Kim, T., Oegema, K., Corbett, K., and Desai, A. (2021). A tripartite mechanism catalyzes Mad2-Cdc20 assembly at unattached kinetochores. Science 371, 64-67. Leontiou, I., London, N., May, K.M., Ma, Y., Grzesiak, L., Medina-Pritchard, B., Amin, P., Jeyaprakash, A.A., Biggins, S., and Hardwick, K.G. (2019). The Bub1-TPR Domain Interacts Directly with Mad3 to Generate Robust Spindle Checkpoint Arrest. Curr Biol 29, 2407-2414 e2407. Musacchio, A. (2015). The Molecular Biology of Spindle Assembly Checkpoint Signaling Dynamics. Curr Biol 25, R1002-1018.

Nijenhuis, W., Vallardi, G., Teixeira, A., Kops, G.J., and Saurin, A.T. (2014). Negative feedback at kinetochores underlies a responsive spindle checkpoint signal. Nat Cell Biol 16, 1257-1264. Nijenhuis, W., von Castelmur, E., Littler, D., De Marco, V., Tromer, E., Vleugel, M., van Osch, M.H., Snel, B., Perrakis, A., and Kops, G.J. (2013). A TPR domain-containing N-terminal module of MPS1 is required for its kinetochore localization by Aurora B. J Cell Biol 201, 217-231.

Overlack, K., Bange, T., Weissmann, F., Faesen, A.C., Maffini, S., Primorac, I., Muller, F., Peters, J.M., and Musacchio, A. (2017). BubR1 Promotes Bub3-Dependent APC/C Inhibition during Spindle Assembly Checkpoint Signaling. Curr Biol 27, 2915-2927 e2917.

Overlack, K., Primorac, I., Vleugel, M., Krenn, V., Maffini, S., Hoffmann, I., Kops, G.J., and Musacchio, A. (2015). A molecular basis for the differential roles of Bub1 and BubR1 in the spindle assembly checkpoint. Elife 4, e05269.

Piano, V., Alex, A., Stege, P., Maffini, S., Stoppiello, G.A., Huis In 't Veld, P.J., Vetter, I.R., and Musacchio, A. (2021). CDC20 assists its catalytic incorporation in the mitotic checkpoint complex. Science 371, 67-71.

Primorac, I., Weir, J.R., Chiroli, E., Gross, F., Hoffmann, I., van Gerwen, S., Ciliberto, A., and Musacchio, A. (2013). Bub3 reads phosphorylated MELT repeats to promote spindle assembly checkpoint signaling. Elife 2, e01030.

Qian, J., Garcia-Gimeno, M.A., Beullens, M., Manzione, M.G., Van der Hoeven, G., Igual, J.C., Heredia, M., Sanz, P., Gelens, L., and Bollen, M. (2017). An Attachment-Independent Biochemical Timer of the Spindle Assembly Checkpoint. Mol Cell 68, 715-730 e715.

Roy, B., Han, S.J.Y., Fontan, A.N., and Joglekar, A.P. (2020). The copy-number and varied strength of MELT motifs in Spc105 balance the strength and responsiveness of the Spindle Assembly Checkpoint. Elife 9.

Roy, B., Han, S.J.Y., Fontan, A.N., and Joglekar, A.P. (2021). Aurora B phosphorylates Bub1 to promote spindle assembly checkpoint signaling. bioRxiv, 2021.2001.2005.425459.

Saurin, A.T., van der Waal, M.S., Medema, R.H., Lens, S.M., and Kops, G.J. (2011). Aurora B potentiates Mps1 activation to ensure rapid checkpoint establishment at the onset of mitosis. Nat Commun 2, 316.

Silio, V., McAinsh, A.D., and Millar, J.B. (2015). KNL1-Bubs and RZZ Provide Two Separable Pathways for Checkpoint Activation at Human Kinetochores. Dev Cell 35, 600-613.

Sullivan, M., Higuchi, T., Katis, V.L., and UhImann, F. (2004). Cdc14 phosphatase induces rDNA condensation and resolves cohesin-independent cohesion during budding yeast anaphase. Cell $117,471-482$.

Taylor, S.S., Hussein, D., Wang, Y., Elderkin, S., and Morrow, C.J. (2001). Kinetochore localisation and phosphorylation of the mitotic checkpoint components Bub1 and BubR1 are differentially regulated by spindle events in human cells. J Cell Sci 114, 4385-4395. 
1012 Vleugel, M., Omerzu, M., Groenewold, V., Hadders, M.A., Lens, S.M., and Kops, G.J. (2015).

1013 Sequential multisite phospho-regulation of KNL1-BUB3 interfaces at mitotic kinetochores. Mol

1014 Cell 57, 824-835.

1015 Vleugel, M., Tromer, E., Omerzu, M., Groenewold, V., Nijenhuis, W., Snel, B., and Kops, G.J.

1016 (2013). Arrayed BUB recruitment modules in the kinetochore scaffold KNL1 promote accurate

1017 chromosome segregation. J Cell Biol 203, 943-955.

1018 Yuan, I., Leontiou, I., Amin, P., May, K.M., Soper Ni Chafraidh, S., Zlamalova, E., and Hardwick, 1019 K.G. (2017). Generation of a Spindle Checkpoint Arrest from Synthetic Signaling Assemblies. Curr 1020 Biol 27, 137-143.

1021 Zhang, G., Lischetti, T., Hayward, D.G., and Nilsson, J. (2015). Distinct domains in Bub1 localize 1022 RZZ and BubR1 to kinetochores to regulate the checkpoint. Nat Commun 6, 7162.

1023 Zhang, G., Lischetti, T., and Nilsson, J. (2014). A minimal number of MELT repeats supports all the 1024 functions of KNL1 in chromosome segregation. J Cell Sci 127, 871-884.

1025 Zhang, G., Mendez, B.L., Sedgwick, G.G., and Nilsson, J. (2016). Two functionally distinct 1026 kinetochore pools of BubR1 ensure accurate chromosome segregation. Nat Commun 7, 12256. 